\title{
Review of the $\mathrm{BCl}$ competition IV
}

\section{Michael Tangermann ${ }^{1}$, Klaus-Robert Müller ${ }^{1,2}$, Ad Aertsen $^{3}$, Niels Birbaumer $^{4,5}$, Christoph Braun ${ }^{6,7}$, Clemens Brunner ${ }^{8,9}$, Robert Leeb ${ }^{10}$, Carsten Mehring ${ }^{3,11,12}$, Kai J. Miller ${ }^{13}$, Gernot R. Müller-Putz ${ }^{8}$, Guido Nolte ${ }^{14}$, Gert Pfurtscheller ${ }^{8}$, Hubert PreissI ${ }^{6,15}$, Gerwin Schalk ${ }^{16,17,18,19,20}$, Alois Schlögl ${ }^{21}$, Carmen Vidaurre ${ }^{1}$, Stephan Waldert ${ }^{3,6,22}$ and Benjamin Blankertz ${ }^{23}$}

${ }^{1}$ Machine Learning Laboratory, Berlin Institute of Technology, Berlin, Germany

${ }^{2}$ Department of Brain and Cognitive Engineering, Korea University, Seoul, Korea

${ }^{3}$ Faculty of Biology, Bernstein Center Freiburg and University of Freiburg, Freiburg, Germany

4 Institute of Medical Psychology and Behavioral Neurobiology, University of Tübingen, Tübingen, Germany

${ }^{5}$ Ospedale San Camillo, Istituto di Ricovero e Cura a Carattere Scientifico, Venezia, Italy

${ }^{6}$ MEG-Center, University of Tübingen, Tübingen, Germany

7 Center of Mind/Brain Sciences, University of Trento, Trento, Italy

${ }^{8}$ Institute for Knowledge Discovery, Graz University of Technology, Graz, Austria

${ }^{9}$ Swartz Center for Computational Neuroscience, Institute for Neural Computation, University of California San Diego, La Jolla, CA, USA

${ }^{10}$ École Polytechnique Fédérale de Lausanne, Lausanne, Switzerland

"Department of Bioengineering, Imperial College London, London, UK

12 Department of Electrical and Electronic Engineering, Imperial College London, London, UK

${ }_{13}$ Physics, Neurobiology and Behavior, Medicine, University of Washington, Seattle, WA, USA

${ }^{14}$ Institute for Neurophysiology and Pathophysiology, University Medical Center Hamburg-Eppendorf, Hamburg, Germany

${ }^{15}$ Department of Obstetrics and Gynecology, University of Arkansas for Medical Sciences, Little Rock, AR, USA

${ }^{16}$ Brain-Computer Interface R\&D Program, Wadsworth Center, New York State Department of Health, Albany, NY, USA

17 Department of Neurology, Albany Medical College, Albany, NY, USA

${ }^{18}$ Department of Neurological Surgery, School of Medicine, Washington University, St. Louis, MO, USA

${ }^{19}$ Department of Biomedical Engineering, Rensselaer Polytechnic Institute, Troy, NY, USA

${ }^{20}$ Department of Biomedical Sciences, School of Public Health, State University of New York, Albany, NY, USA

${ }^{21}$ Institute for Science and Technology Austria, Maria Gugging, Austria

${ }^{22}$ Sobell Department of Movement Neuroscience and Movement Disorders, Institute of Neurology, University College London, London, UK

${ }^{23}$ Neurotechnology Group, Berlin Institute of Technology, Berlin, Germany

\section{Edited by:}

Eilon Vaadia, The Hebrew University, Israel

\section{Reviewed by:}

Kenji Kansaku, Research Institute of National Rehabilitation Center for Persons with Disabilities, Japan Jose L. "Pepe" Contreras-Vidal, University of Houston, USA

\section{*Correspondence:}

Michael Tangermann, Machine

Learning Laboratory, Berlin Institute of Technology, FR 6-9, Franklinstr.

28/29, 10587 Berlin, Germany.

e-mail: michael.tangermann@

tu-berlin.de
The $\mathrm{BCl}$ competition IV stands in the tradition of prior $\mathrm{BCl}$ competitions that aim to provide high quality neuroscientific data for open access to the scientific community. As experienced already in prior competitions not only scientists from the narrow field of $\mathrm{BCl}$ compete, but scholars with a broad variety of backgrounds and nationalities. They include high specialists as well as students. The goals of all $\mathrm{BCl}$ competitions have always been to challenge with respect to novel paradigms and complex data. We report on the following challenges: (1) asynchronous data, (2) synthetic, (3) multi-class continuous data, (4) sessionto-session transfer, (5) directionally modulated MEG, (6) finger movements recorded by ECoG. As after past competitions, our hope is that winning entries may enhance the analysis methods of future $\mathrm{BCls}$.

Keywords: brain-computer interface, $\mathrm{BCl}$, competition

\section{INTRODUCTION}

Brain-computer interfacing (BCI) is an approach to establish a novel communication channel from men to machines. The crucial idea is to directly tap the communication at its very origin: the human brain. BCI technology is used to date primarily for intentional control. This branch of BCI research aims at the (partial) restoration and rehabilitation of lost functions in paralyzed patients (Kübler et al., 2001; Wolpaw et al., 2002). The focus of the fourth BCI competition was on BCI systems that are based on the motor and sensorimotor system of the brain. In line with the past three BCI competitions, this fourth BCI competition strives to help the field of $\mathrm{BCI}$ prosper by eliciting solutions for hard data analysis problems appearing in current BCI research.
Apart from communication and control, recently more and more alternative applications of $\mathrm{BCI}$ technology are being explored (Blankertz et al., 2010). These include enhancement of human performance (Haufe et al., 2011) and assessing subconscious perception (Porbadnigk et al., 2010, 2011). Data from those recent developments have not yet been included in the BCI competitions, but may pose interesting and novel challenges for future competitions.

\subsection{RELEVANCE OF BCI COMPETITIONS}

The impact of the past three competitions on the field of BCI research is manifold and thus worth a closer look. One indicator of the overall relevance of the BCI competitions for the scientific community is the number of citations. Figure 1 shows how 


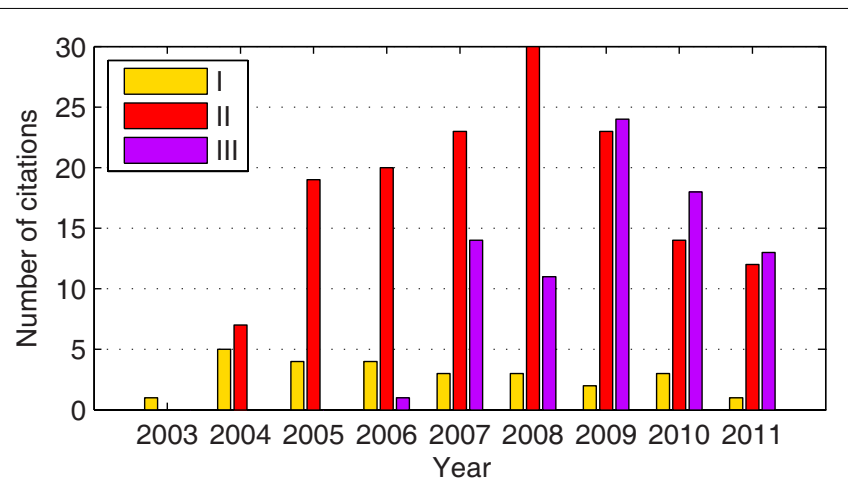

FIGURE 1 | Citations of the overview articles on previous competitions. The histogram shows how many times the editorial articles on BCl competitions I (Sajda et al., 2003), II (Blankertz et al., 2004), and III (Blankertz et al., 2006) have been cited in ISI-indexed journals. Data were retrieved from the ISI Web of Knowledge on December 1st 2011.

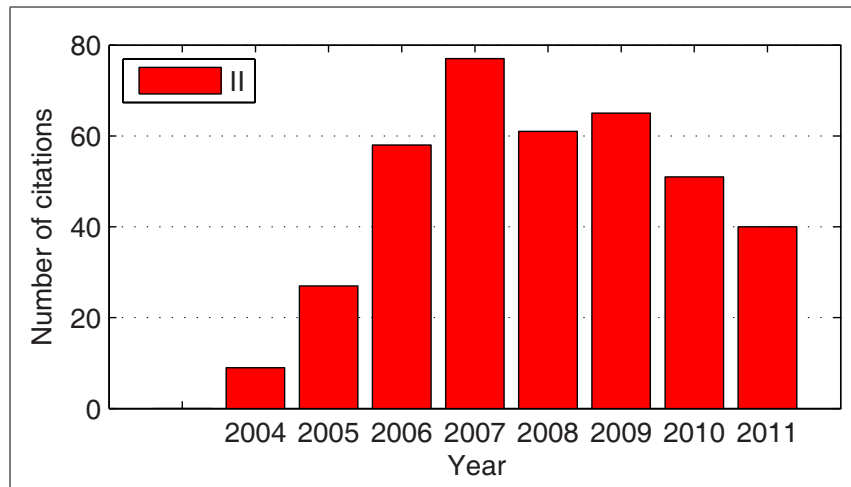

FIGURE 2 | Citations of the articles by the competition winners. The histogram shows how many times the articles of the winning teams of $\mathrm{BCl}$ competition II (describing the winning algorithms) have been cited in ISI-indexed journals. Data were retrieved from the ISI Web of Knowledge on December 1st 2011.

often the three overview articles on the past BCI competitions I (Sajda et al., 2003), II (Blankertz et al., 2004), and III (Blankertz et al., 2006) have been cited in ISI-indexed journals and conference proceedings. The overall sum is 255. From competition II on, the concept was introduced to have publications of all winning algorithms within one issue of a journal. This worked very well in the BCI competition II where all winner articles have been published in volume 51 of IEEE Trans Biomed Eng (Blanchard and Blankertz, 2004; Bostanov, 2004; Kaper et al., 2004; Lemm et al., 2004; Mensh et al., 2004; Wang et al., 2004; Xu et al., 2004). Such concerted publication leads to good visibility, and as a consequence to substantial citations, see Figure 2. (In competition III only some winning algorithms were published spread cross several journals; Wei et al., 2006; Galan et al., 2007; Zhang et al., 2007; Rakotomamonjy and Guigue, 2008.)

Moreover, research groups that are relatively new to the field of BCI can attract attention and get renowned if the performance of their algorithms is independently validated through the competition process. This is an attractive opportunity even for researchers who do not have access to an acquisition device for brain signals or a fully running BCI system. Additionally, some researchers of the better performing teams were hired or hosted by BCI groups (in particular the one contributing data sets to the competition).

Most important, the results of the BCI competitions provide an indication of what type of methods are effective. A good example of such a lesson that can be learned from the competitions is that common spatial pattern analysis (CSP/CSSD; Koles, 1991; Ramoser et al., 2000; Blankertz et al., 2008b) and its variants are a robust tool for exploiting ERD/ERS effects (Pfurtscheller and da Silva, 1999): Almost all data sets throughout all BCI competitions in which CSP was reasonably applicable (e.g., for multi-channel recordings or for paradigms in which differential ERD/ERS effects are expected) have been won by an algorithm involving a variant of CSP: competition II (2a, 4); competition III (1, 3, 4a, 4c); competition IV (1, 2a, 2b). The success of the CSP-based methods in the BCI competitions may have a promoting factor for the flourishing development of variants of CSP analysis (Lotte and Guan, 2011; Nikulin et al., 2011; Sannelli et al., 2011).

In contrast, the application of principle component analysis (PCA) or independent component analysis (ICA), which are very successful preprocessing methods in other application fields, seem to be a less effective ingredient to improve the classification performance in BCI (but note, that ICA was used in Xu et al., 2004). This advance of CSP compared to PCA and ICA may to a large extend be explained by the different strategies concerning the use of class labels. While CSP exploits the information contained in the labels in a supervised manner, ICA and PCA are unsupervised methods.

In this context, we would like to stress that the competitions are by no means a systematic evaluation of all available algorithms. Therefore, we would still like to encourage to explore the full realm of signal processing and pattern recognition algorithms for BCI.

\subsection{THE ROLE OF OPEN DATA}

$\mathrm{BCI}$ research is complex, and to design an online BCI experiment or successfully run a BCI application involves the cooperation of specialists from various disciplines. The availability of BCI data from past competitions is an important contribution to stimulate the interdisciplinary engagement of students and researchers from neighboring research areas, who can enrich the field of BCI. This is especially true for scientists specialized in signal processing, data analysis, and machine learning, but also for researchers from the field of human-computer interaction (HCI). While these specialists have the potential to improve the progress of BCI with new algorithmic methods or improved usability of BCI applications, the field of BCI needs to provide the fuel, that is data. Data, that on the one hand is typically noisy, high-dimensional, shows non-stationary characteristics, and thus provides a challenging test ground especially for the signal processing and machine learning community. On the other hand, BCI data represents - if interpreted as a signal for communication and control - an inherently unreliable and slow communication channel. From the viewpoint of HCI, the field of BCI can be considered a challenge as it requires highly robust interaction models in order to cope with the above 
mentioned challenges. Finally, any success story for the interaction design in BCI might be transferable into the other fields like usability of mobile devices or gesture controlled applications, which share some of these interesting characteristics.

\subsection{NOTES ON THE USE OF BCI-COMPETITION DATA}

Despite of a number of high quality algorithmic solutions proposed by the competition winners in the following sections, the actual learning problems posed in this competition are surely of interest in the future, and the proposal of new methods for their solution can enhance the field of BCI. For this reason, the competition data sets have been provided online as open data. Furthermore, the labels of the test data, which have not been available to the participants of the BCI competition IV, have been published in addition.

We would like to encourage the use of this data and the publications of any results and insights. Upon publication of such results, however, we would like to draw your attention to three important aspects:

First, any performance improvement over the competition results, should be reported with a note of caution, as it could merely reflect random fluctuations. Ideally, the performance should be reported for larger amounts of test data.

Second, a comparison with the performance of the competitors should be drawn carefully only, as any post-competition work on the same data has been performed under the advantage of knowing the competition outcome, knowing the specific shortcomings of the submitted algorithms, and having insight into which classes of algorithms perform better or worse on that data.

Third, even so the test data labels are publicly available now, their use should be restricted to finally determine the performance of a method. The test data should not be touched at all during the algorithm design process and the determination of hyperparameters, as this can lead to a substantial amount of overfitting (Lemm et al., 2011).

\subsection{RELEVANCE OF THE DATA SETS}

For an overview, a list of data sets and the corresponding winning teams is summarized in Tables $\mathbf{1}$ and $\mathbf{2}$.

The BCI competition fosters algorithmic solutions, which allow for a single-trial assessment of mental states. For the neurosciences, such developments in signal processing and machine learning are

Table 1 | Overview of the data sets of $\mathrm{BCl}$ competition IV.

\begin{tabular}{|c|c|c|c|}
\hline \# & Lab & \# Channels & Paradigm and challenge \\
\hline 1 & Berlin & 64 EEG & $\begin{array}{l}\text { 2-Class motor imagery, uncued } \\
\text { classifier application }\end{array}$ \\
\hline $2 a$ & Graz & $22 \mathrm{EEG}$ & $\begin{array}{l}\text { 4-Class motor imagery, continuous } \\
\text { classifier application }\end{array}$ \\
\hline $2 b$ & Graz & 3 EEG & $\begin{array}{l}\text { Motor imagery, session-to-session } \\
\text { transfer and eye artifacts }\end{array}$ \\
\hline 3 & Freiburg & $10 \mathrm{MEG}$ & $\begin{array}{l}\text { Decoding directions of } \\
\text { finger/hand/wrist movements }\end{array}$ \\
\hline 4 & Seattle/Albany & $64 \mathrm{ECoG}$ & $\begin{array}{l}\text { Discrimination of movements of } \\
\text { individual finders }\end{array}$ \\
\hline
\end{tabular}

clearly relevant as these single-trial data analysis methods provide a possibility to monitor the acting and behaving brain. This is a prerequisite to study the dynamics of brain processes, and eventually develop new reactive experimental paradigms, that vary, e.g., stimulus conditions depending on the current state in a closed loop.

The data sets of this competition all deal with motor paradigms, and more specifically with oscillatory signals which are related to imagined motor actions or motor execution. As an example, direct clinical relevance of BCI technology can be expected for the support of rehabilitation training in patients suffering from stroke (Silvoni et al., 2011) in cortical motor areas. However, as changes of oscillatory processes are not uniquely observed during motor activities, but represent a rather general high-level characteristic of many brain processes, the benefit of this BCI competition should extend from motor system research to other fields.

Data set 1 of the BCI competition IV addresses the challenge to correctly deal with intended non-control periods and uncued periods of control activity. This is of high clinical relevance, as any practical application of a motor imagery BCI system will require that the BCI system recognizes periods of resting and coming back to active BCI control.

Data set $2 \mathrm{a}$ enlarges the number of control classes from two to four. Compared to the simpler setting of only two motor imagery classes, this enlargement contains the risk of a reduction in classification accuracy. However, it also offers the potential of higher information transfer rates, and more natural interaction paradigms between user and application. In combination with the continuous classification setting, this is clearly of practical relevance.

Data set $2 \mathrm{~b}$ challenges the session-to-session transfer of classification models. Avoiding the time-consuming re-calibration of the BCI system, such approaches are of high practical importance for end-users, who want to use a BCI on a daily basis.

Data set 3 is a collection of magnetoencephalography (MEG) signals. While most motor paradigms in non-invasive BCI make use of the lateralization of motor-related signals (e.g., ERD/ERS effects over the left hand and right hand cortex), this data set seeks to extract a multi-class decision from a single hand only. Comparable to data set $2 \mathrm{a}$, the expansion from two to more classes has the potential to boost the information transfer rate of a BCI. Furthermore the data set is an example for the possibility to infer hand movement directions not only from single cell spiking activity (e.g., by intra-cortical single unit recordings; Georgopoulos et al., 1982; Velliste et al., 2008), which are known to realize a directional coding, but also from non-invasive measurements of larger populations (Waldert et al., 2008). Despite of its practical restrictions (an MEG system is neither practical nor affordable for patients), this MEG-BCI could still be applied in conjunction with online feedback, e.g., for stroke rehabilitation attempts (Buch et al., 2008) or for prosthesis training.

The goal for data set 4 of the BCI competition IV was to infer the flexion of individual fingers from signals recorded from the surface of the brain via electrocorticography (ECoG). Determining the relationship of ECoG signals with finger flexion provides new neuroscientific understanding, and may eventually lead to improved brain-computer interface systems. 
Table 2 |This Table lists the winning teams for all competition data sets.

\begin{tabular}{|c|c|c|}
\hline Data set & Research lab & Contributor(s) \\
\hline 1 & Institute for Infocomm Research, Singapore & $\begin{array}{l}\text { Zhang Haihong, Ang Kai Keng, Guan Cuntai, Wang Chuanchu, Chin } \\
\text { Zheng Yang }\end{array}$ \\
\hline $2 a$ & Institute for Infocomm Research, Singapore & $\begin{array}{l}\text { Kai Keng Ang, Zheng Yang Chin, Chuanchu Wang, Cuntai Guan, } \\
\text { Haihong Zhang, Kok Soon Phua, Brahim Hamadicharef, Keng Peng } \\
\text { Tee }\end{array}$ \\
\hline $2 b$ & Institute for Infocomm Research, Singapore & $\begin{array}{l}\text { Zheng Yang Chin, Kai Keng Ang, Chuanchu Wang, Cuntai Guan, } \\
\text { Haihong Zhang, Kok Soon Phua, Brahim Hamadicharef, Keng Peng } \\
\text { Tee }\end{array}$ \\
\hline 3 & $\begin{array}{l}\text { Biomedical Signal and Image Processing Laboratory (BiSIPL), Sharif } \\
\text { University of Technology, Tehran, Iran }\end{array}$ & Sepideh Hajipour, Mohammad Bagher Shamsollahi \\
\hline 4 & Cortex Team, Research Centre INRIA, France & Nanying Liang, Laurent Bougrain \\
\hline
\end{tabular}

\subsection{OVERVIEW OF THE ARTICLE}

After some general remarks concerning the concept and the BCI competitions in Section 2, the subsequent five sections, will characterize each data set contained in the BCI competition IV in detail, including an assessment of its relevance to the field, experimental details, the data format, the applied evaluation criterion for submissions, and a brief outcome. The article closes with a section about the overall results of the competition and a discussion. The latter includes prospective topics of subsequent competitions.

The winning labs published individual articles on their approaches, see (Ang et al., 2012; Flamary and Rakotomamonjy, 2012; Sardouie and Shamsollahi, 2012; Zhang et al., 2012).

\section{GENERAL STRUCTURE OF THE DATA SETS AND THE MACHINE LEARNING TASK}

Challenges posed within the BCI competition typically contain a problem description, a training data set, a test data set, and a description of the evaluation metric that is applied to determine the performance of contributed algorithms.

\subsection{TRAINING DATA}

This collection of data (also called calibration data) comprises the data epochs from EEG, MEG, or ECoG recordings, the labels or markers that describe the tasks that were to be performed by subjects at recording time, and the cues which had been presented to them. In addition, the groups providing the training data describe the specific performance metric according to which any participant's competition entry will be rated. Participants used this information to develop a processing method that was able to estimate labels based on data. Any method could only be successful, if it generalized well on new test data.

\subsection{TEST DATA}

This data set (also called evaluation data) contains data epochs, but no labels or markers. The labels do exist but were secret to the participants. The task of participants was to estimate the labels of the test data and send them in. The data providing group evaluated the labels according to the predefined performance metric, that had been published together with the training data.

\subsection{CAUSALITY OF METHODS}

As the full test set is available to the participants from the beginning and not (as in a real online experiment) incrementally, the participants could in principle exploit the structure of the full (unlabeled) data already in advance in order to improve their label estimate even for the first trials. The organizers are aware of the problem, that this use of data is non-causal and unrealistic. Consequently it was not allowed for participants to exploit this unrealistic advantage, that they could gain compared to a BCI practitioner.

However, the distribution of test data is simplified to a large extend, if it can be provided en bloc. In order to ensure causal processing despite of this distribution method, the participants had to submit a short description of the developed data processing routines. In case of unclear causality the participants had to prove that their approach is causal by handing in the data processing routines in addition to the labels.

\section{DATA SET 1}

Data set 1 Asynchronous Motor Imagery is provided by B. Blankertz, C. Vidaurre and K.-R. Müller from Berlin (Germany). It can be freely assessed via http://www.bbci.de/competition/iv/with the only restriction that the present article is referenced upon any publication of results.

\subsection{MOTIVATION}

Most demonstrations of algorithms on BCI data are evaluating classification of EEG trials, i.e., segments of EEG signals of a fixed length, where each trial corresponds to a specific mental state. But in BCI applications with asynchronous feedback, e.g., cursor control, one is faced with the problem that the classifier has to be applied continuously to the incoming EEG without having cues of when the subject is switching her/his intention. This data set poses the challenge of applying a classifier to continuous EEG for which no cue information is given.

Another issue that is addressed in this data set is that the test data contains periods in which the user has no control intention. During those intervals the classifier is supposed to return to 0 (no affiliation to one of the target classes).

As a special feature, some of the data sets were artificially generated. The idea was to have a means for generating artificial EEG 
signals with specified properties that are so realistic that they can be used to evaluate and compare analysis techniques. The competition is a possibility to verify whether the applied methods perform comparably on artificial and real data. The only information provided to the competitors was that there is at least one real and at least one artificial data set, while the true distribution remained undisclosed until the submission deadline. For competition purpose, only results for the real data set(s) were considered, but results for artificial data were also reported for comparison. See the subsequent Section 4 for a detailed description of the generation of the artificial data and a comparison of the competition results obtained on real vs. artificial data.

\subsection{MATERIALS AND SUBJECTS}

These data sets were recorded exclusively for the purpose of the competition. Four healthy participants served as experimental subjects. In the whole session motor imagery was performed without feedback. For each participant two classes of motor imagery were selected from the three classes left hand, right hand, and foot ( side chosen by the individual; optionally also both feet).

\subsubsection{Experimental paradigm}

The recording was made using BrainAmp $M R$ plus amplifiers (Brain Products $\mathrm{GmbH}$, Munich, Germany) and a Ag/AgCl electrode cap (EASYCAP GmbH). Signals from 59 EEG positions were measured that were most densely distributed over sensorimotor areas. Signals were band-pass filtered between 0.05 and $200 \mathrm{~Hz}$ and then digitized at $1000 \mathrm{~Hz}$ with 16 bit $(0.1 \mu \mathrm{V})$ accuracy. Also a version of the data was provided that was sub sampled at $100 \mathrm{~Hz}$ [first low-pass filtering the original data (Chebyshev Type II filter of order 10 with stop band ripple $50 \mathrm{~dB}$ down and stop band edge frequency $49 \mathrm{~Hz}$ ) and then calculating the mean of consecutive blocks of 10 samples].

\subsubsection{Protocol}

The session was divided into two parts: recording of training data and recording of test data. Training data were provided with complete marker information such that it could be used by the competitors for adapting the parameters of the methods/models. In contrast, the test data which was provided to the competitor only consisted of the EEG signals. The corresponding markers have been kept secret until the submission deadline and have been used to evaluate the submissions.

3.2.2.1. Training data. In the first two runs, arrows pointing left, right, or down were presented as visual cues on a computer screen. Cues were displayed for a period of $4 \mathrm{~s}$ during which the subject was instructed to perform the cued motor imagery task. These periods were interleaved with $2 \mathrm{~s}$ of blank screen and $2 \mathrm{~s}$ with a fixation cross shown in the center of the screen. The fixation cross was superimposed on the cues, i.e., it was shown for $6 \mathrm{~s}$, see Figure 3. In each run 50 trials of each of the chosen two classes have been presented, resulting in a total of 200 trials. After every 15 trials a break of $15 \mathrm{~s}$ was given for relaxation. Between the runs there were longer breaks of 5-15 min.

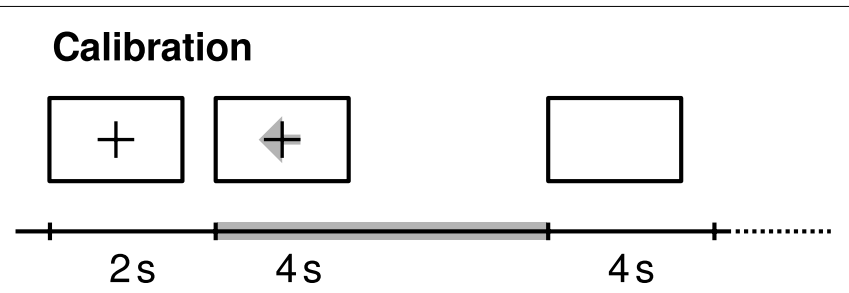

\section{Evaluation}

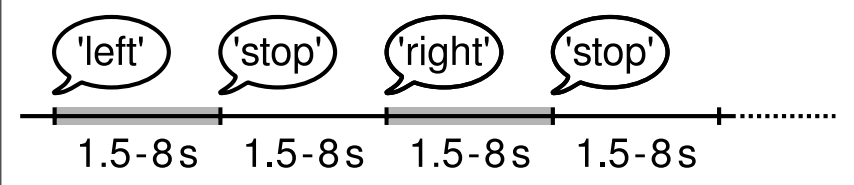

FIGURE 3 | (Data set 1 - trial structure). Training data was collected in the calibration runs. Arrows pointing left, right, or down have been presented as cues for imagining left hand, right hand, or foot movements. After a fixation cross was presented for $2 \mathrm{~s}$, the directional cue was overlaid for $4 \mathrm{~s}$. Then the screen was blank for $2 \mathrm{~s}$. In the test runs used for evaluation, spoken words have been presented as cues.

3.2.2.2. Test data. Then 4 runs followed which were used for evaluating the submissions to the competitions. Here, the motor imagery tasks were cued by acoustic stimuli (words left, right, and foot) for periods of varying length between 1.5 and $8 \mathrm{~s}$. The end of the motor imagery period was indicated by the word stop. Intermitting periods had also a varying duration of $1.5-8 \mathrm{~s}$. The acoustical cues were soft-spoken it order to avoid that acoustically evoked potentials could be detected to segment the data into control and no-control intervals (or even to decode the cue information). In each run, 30 trials for each class have been recorded resulting in a total of 240 trials. After every 30 trials a break of $15 \mathrm{~s}$ was given for relaxation. Between the runs there were longer breaks of 5$15 \mathrm{~min}$. Competitors were informed that the number of trials from each condition was not necessarily equal. Due to the experimental design, there were twice as much periods of no control as periods of each condition.

Additionally, we introduced a kind of non-stationarity into the test data by changing the environmental conditions. Occasionally during the runs music (2 times) or videos (2 times) have been played, or the participant was instructed to close her/his eyes (2 times). Each of those periods (during which the cue presentation was not paused) lasted about $2 \mathrm{~min}$.

\subsection{INVESTIGATION OF THE DATA SET}

The most stable effect of motor imagery is a modulation of the sensorimotor rhythms (SMRs), see (Pfurtscheller and da Silva, 1999). For hand motor imagery an attenuation of the SMR amplitude over the contralateral motor area is expected. The effect of foot imagery is more diverse. An attenuation of the SMR over the foot area, which is on the midline of the motor cortex could be expected, but is rarely observed and does not appear in the data set. In most of the subjects, an increase of the SMR amplitude over the hand areas is observed. This is also the case for the two participants ( $a$ and $f$ ) of this data set, who performed foot imagery. Figure 4 gives an overview, of how this effect is reflected in the 


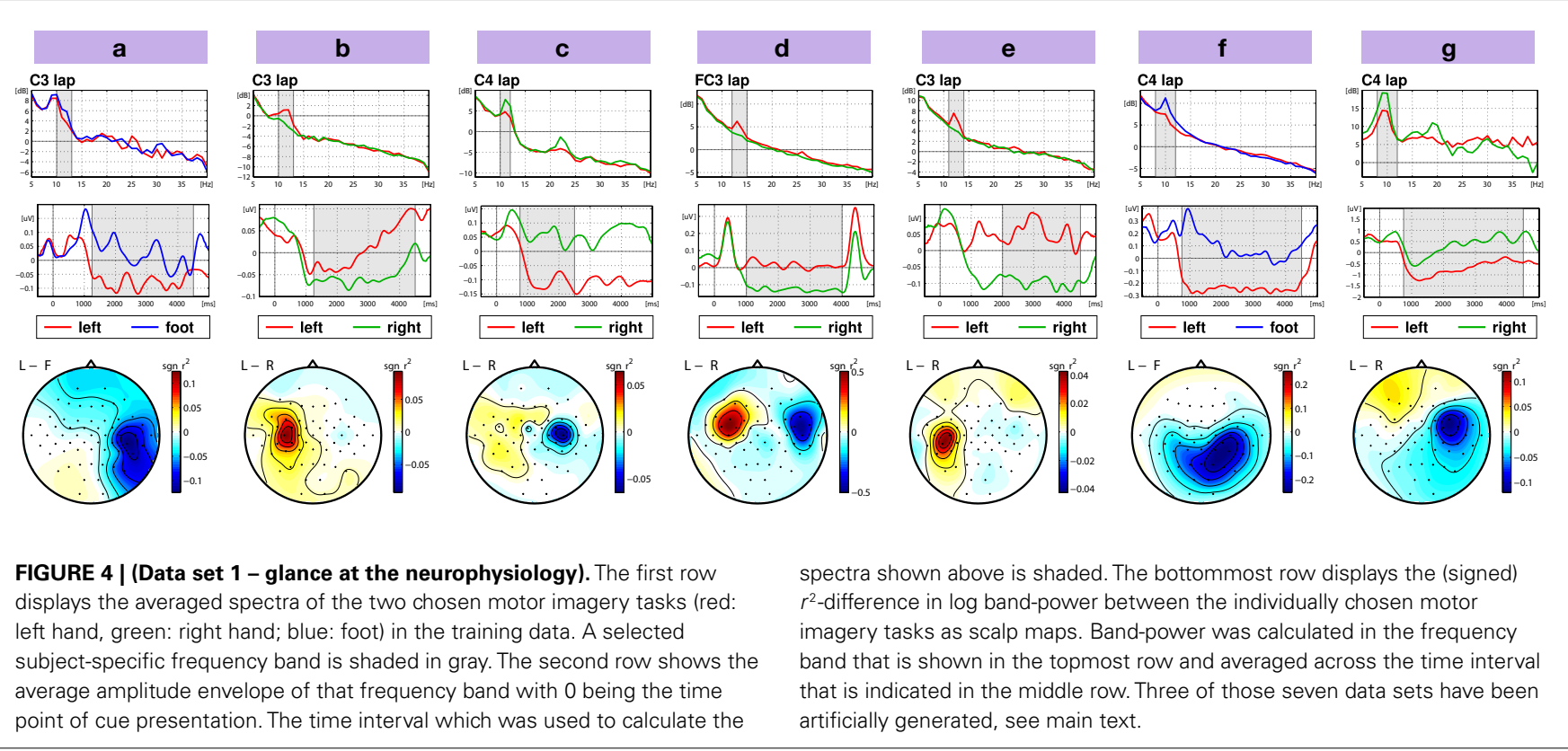

competition data set. For each participant, an individual channel, time interval, and frequency band was selected to display the differential modulations of the SMRs. Class-wise averaged frequency spectra are plotted in the upper row. The second row shows the time course of band-power averaged across all trials. The bottommost row displays the difference in log band-power between the two motor imagery conditions as scalp topographies.

Data sets $c, d$, and $e$ were artificially generated.

\subsection{Challenge}

The submissions were evaluated in view of a one dimensional cursor control application with range from -1 to 1 . The mental state of class one is used to position the cursor at -1 , and the mental state of class two is used to position the cursor near 1 . In the absence of those mental states (intermitting intervals) the cursor should be at position 0 . Note that it is unknown to the competitors at which intervals the subject is in a defined mental state. Competitors had to submit classifier outputs for all time points. To measure the performance, the squared error with respect to the target vector - that is -1 for class one, 1 for class two, and 0 otherwise - averaged across time points was calculated. Since the mental state of the user does not abruptly change with cue appearance, time points during transient periods ( $1 \mathrm{~s}$ starting from each cue) were discarded from evaluation.

As stated above, it was declared that for competition purpose, only results for the real data sets were considered, but results for artificial data were also reported for comparison.

Additionally, participants were asked to optionally judge which of the data sets were the artificially generated ones.

\subsection{OUTCOME IN BRIEF}

There were 24 submissions to data set 1 . The winning team is Zhang Haihong and colleagues from the Institute for Infocomm Research, Singapore. They approached the task as a three class problem with the rest class being the third class. For classification,
CSP was combined with a filter bank. A criterion based on mutual information was used to select those features that were to be fed into a radial basis function based neural network. Using this approach, they obtained a mean squared error (MSE) of 0.382 (averaged across the four real data sets). For further details of their method see (Zhang et al., 2012). The winners are very closely followed by Dieter Devlaminck and colleagues from the University of Ghent, from the Psychiatric Institute of Guislain and from the University Hospital Ghent, who obtained an MSE of 0.383 . They employed multi-class CSP with a subject-specific frequency band and a multi-class support vector machine (SVM) with ordinal regression. The results ranked 3 rd to 5 th have been achieved by Kai Keng Ann and colleagues (Institute for Infocomm Research, Singapore); Liu Guangquan and colleagues (Shanghai Jiao Tong University, China), and Abdul Satti and colleagues (University of Ulster). All those three competitors also used CSP as a pivotal step in combination with a filter bank (rank 3 ) or with a subject-specific frequency band (ranks 4 and 5). Figure 5 shows histograms of the results of those five highest ranked submissions.

To assess the results, it has to be taken into account that a classifier that gives the constant output zero has an MSE of about 0.5. The exact value varies between data sets since the length of the motor imagery and no-control period was chosen randomly. Figure 6 gives a more detailed view on the performance of the winning algorithm. It shows for the four data sets (rows) normalized histograms of the classifier outputs - separately for periods of the three mental states. In the left column the true label is -1 (first motor imagery class), in the middle column the true label is 0 (no control intention), and in the right column the true label is 1 (second motor imagery condition). The value of the true label is indicated by a blue triangle in each subplot. This figure makes clear that this data set poses really a big challenge. Even for the best method among 24 submissions, the results are not very satisfying. Interestingly, the no-control 
state is quite well detected in the second data set (participant $b$ ). The overall best performance was achieved in the forth data set (participant $g$ ).

Figure 7 gives a better intuition of how well the obtained control actually is. It shows for a selected segment of $100 \mathrm{~s}$ the true

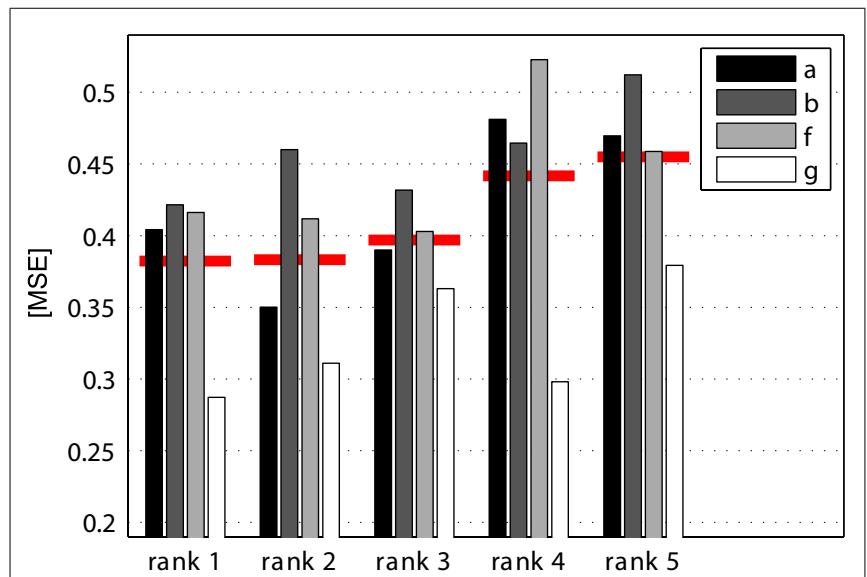

FIGURE 5 | (Data set 1 - histogram of results). Performance of the first five ranked submissions is shown in terms of their mean squared error (MSE) wrt. the true labels. Only results for the real (i.e., not artificially generated) data sets are shown. The mean across the four data sets is plotted as a horizontal red line. The MSE for constant prediction output of 0 are $0.507,0.515,0.491,0.524$ for data sets $a, b, f, g$, respectively. mental state (blue bars) and the classifier output of the winning algorithm (red line).

A guess on the question which data sets were artificially generated was submitted by 16 out of 23 competitors. The correct categorization was revealed by two competitors (Astrid Zeman and Manuel Moebius), 8 more competitors revealed 2 of the 3 artificial subjects, but one of those also considered one real data set as artificial.

\section{DATA SET 1 (ARTIFICIALLY GENERATED)}

The subset of Data set 1 that was artificially generated is provided by C. Vidaurre and G. Nolte from Berlin (Germany). It can be freely assessed via http://www.bbci.de/competition/iv/ with the only restriction that the present article is referenced upon any publication of results.

\subsection{MOTIVATION}

The BCI competition IV included an original ingredient compared to past events: part of the data sets of the BBCI group were artificially generated. The motivation of this work was to check whether or not EEG data can be created to have specific properties in order to test new machine learning methods. If this was the case, the algorithms applied to both, synthetic and real EEG, would produce comparable results. To test this hypothesis we analyzed the ranking of the participants and the performance of the methods in real and synthetic EEG. Brain data like EEG is often noisy and its variables cannot be controlled easily. Synthetically generated data
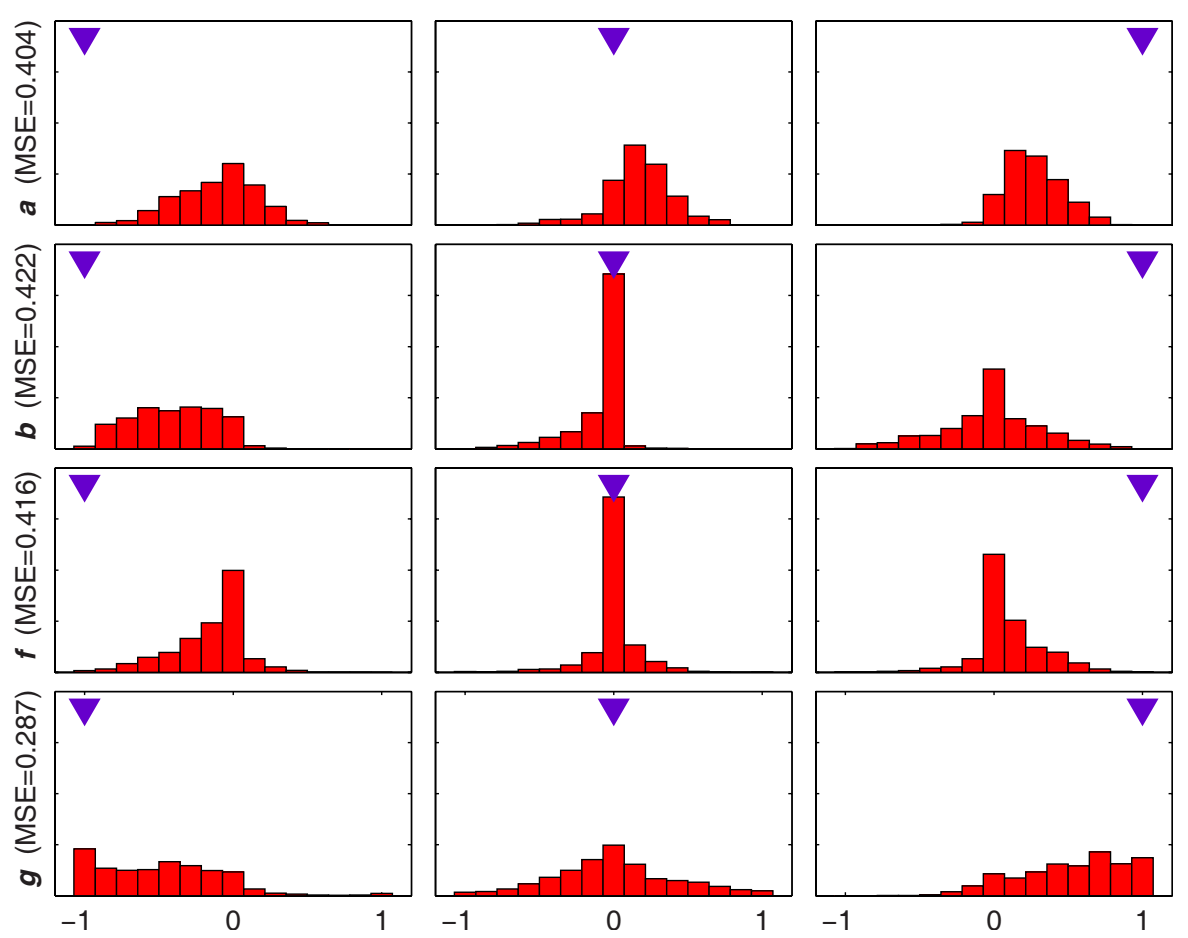

FIGURE 6 | (Data set 1 - distribution of classifier outputs). These (normalized) histograms display the distribution of the classifier outputs of the winning algorithm. Each row corresponds to one data set $(a, b, f, g)$. The left column is a histogram for those time points in which the true label is -1 , for the middle column it is 0 (no control), and for the right column it is 1 . The true label is indicated by the blue triangle. 


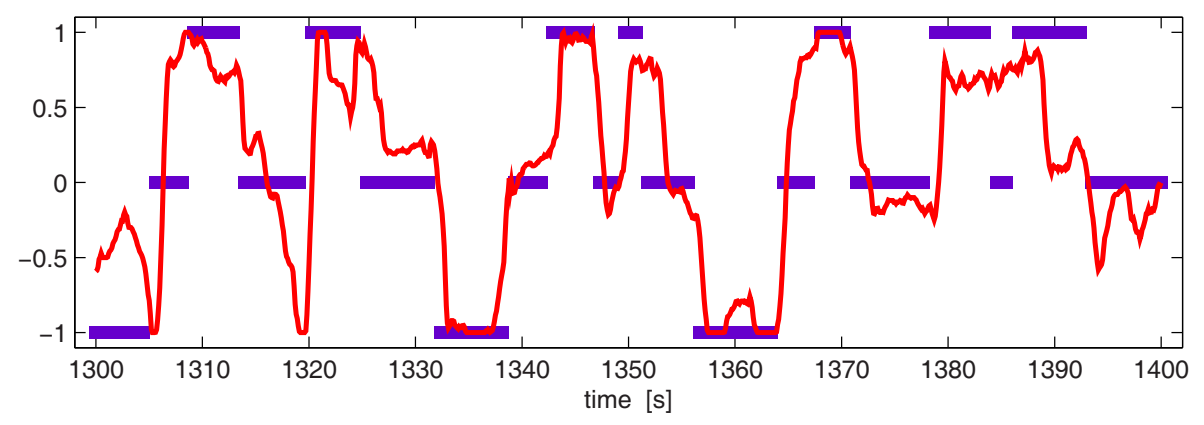

FIGURE 7 | (Data set 1 - trace of classifier outputs). The labels of the true mental state are displayed in blue. The red line shows the classifier outputs of the winning algorithm. This example is a selected segment of $100 \mathrm{~s}$ taken from data set $g$ in which the classification is quite successful. The MSE in the shown segment is 0.171 . may overcome these difficulties, and besides it is easy and cheap to produce.

\subsection{MATERIAL}

In the following, the single components of (artificial) EEG are described separately. We start with the generation of artificial EEG noise, which we divided into background noise and baseline drifts. Then we describe the generation of the $\mu$ (and $\beta$ as a first harmonic of $\mu$ ) rhythm and its desynchronization (ERD) due to the onset of motor imagery tasks. After that, we describe the artifacts that have been added to the data (eye blinks and eye movements) to include some more realistic noise in our signals. Our synthetic EEG is computed as a superposition of potentials from these three different systems (ongoing background noise, task dependent rhythmic activity generated on the motor cortex and eye related noise, eye blinks, and eye movements) which have qualitatively different statistical and spatial properties.

\subsubsection{Background noise}

Background noise in EEG can reasonably be assumed to be Gaussian distributed. The spatial and temporal characteristics, however, are in general too complex to be adequately modeled using a simple parametric model (Huizenga et al., 2002; Bijma et al., 2003; Freeman, 2004a,b, 2005, 2006). To solve this difficulty, we first estimated the cross-spectrum from real EEG data in eyes open and eyes closed conditions and then generated an arbitrary amount of data according to the estimated (complex) cross-spectral matrix in the following way:

Let $x_{i}(f)$ be the Fourier transform of simulated white Gaussian noise for $N$ data points for channel $i$ with $i=1$...M. Since typically (and in the case of our data) the estimated cross-spectrum $C\left(f_{i}\right)$ at discrete frequencies $f_{i}$ is based on averages of relatively short time windows (duration: $1 \mathrm{~s}$ ) and $N$ denotes the length of the complete data set, the frequency resolution of the measured cross-spectra is much lower than the frequency resolution of $x_{i}(f)$. We estimate $C(f)$ as a linear interpolation:

$C(f) \equiv=\frac{f-f_{1}}{f_{2}-f_{1}} C\left(f_{1}\right)+\frac{f_{2}-f}{f_{2}-f_{1}} C\left(f_{2}\right)$

with $f_{1}\left(f_{2}\right)$ being the largest (smallest) value of the set $\left(f_{i}\right)$ lower (higher) than $f$. Then we scale $\mathbf{x} \equiv\left(\mathbf{x}_{1}(\mathbf{f}), \mathbf{x}_{\mathbf{2}}(\mathbf{f}), \ldots, \mathbf{x}_{\mathbf{M}}(\mathbf{f})\right)^{\mathbf{T}}$ with $\mathrm{A}(f)$ defined by the decomposition ${ }^{1} C(f)=A(f) A(f)^{\dagger}$ with ${ }^{\dagger}$ denoting transpose and complex conjugation:

$\mathbf{y}(\mathbf{f})=\mathbf{A}(\mathbf{f}) \mathbf{x}(\mathbf{f})$

Finally, the simulated noise data in the channel $i$ is calculated as the inverse Fourier transform of $y_{i}(f)$. The resulting background noise was a superposition of different amounts of each type of noise, depending on the condition. Figure 8 depicts noise in conditions eyes open and eyes closed at the $10-\mathrm{Hz}$ frequency. One can observe that the power at this frequency is varying in the occipital region, as expected in real EEG data.

\subsubsection{Baseline drifts}

Baseline drifts are typically observable in unfiltered electroencephalographic signals (cf. Simons et al., 1981; Henninghausen et al., 1993) and this is also the case for the BCI-competition data. After analyzing the real "raw" EEG of the competition, we observed both, relatively fast and slow drifts of the signal (shown in Figure 9) and accordingly created two types of artificial drifts. These drifts were generated using the cross-spectrum of the background noise, but simulating a higher sampling frequency, which had the effect of producing a slower signal (noise) than the background itself. The selected frequencies for this computation were 150 and $300 \mathrm{kHz}$, respectively (the original sampling frequency was $1 \mathrm{kHz}$ ).

\subsubsection{Event-related desynchronization}

Forward calculation For the generation of ERD we assumed fixed spatial patterns calculated as potential maps from dipolar sources within left and right motor areas (Geselowitz, 1967). Again, we assumed that the data is Gaussian distributed. The frequency content, however, was restricted to a single frequency (chosen to be $12 \mathrm{~Hz}$ ) with a width $\delta f=1 \mathrm{~Hz}$. We assumed that the generators of this rhythm were radial dipoles with the origins to be $3 \mathrm{~cm}$ below electrodes $\mathrm{C} 3$ and $\mathrm{C} 4$ for the left and right side activity, respectively (see Figures 10 and 11). The directions "radial" and also "below" were chosen according to the surface normals at electrodes C3 and C4. For the forward calculation we assumed a realistic volume conductor consisting of three shells (scalp, skull, brain)

${ }^{1}$ The decomposition is not unique but any will do. 


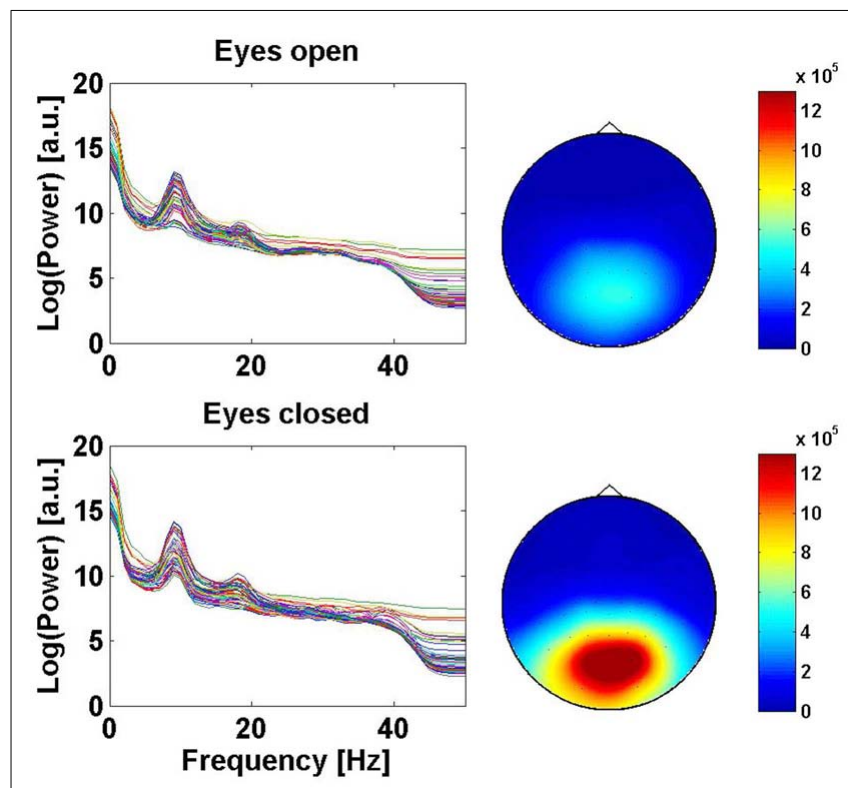

FIGURE 8 | (Data Set 1 - artificial). Left: spectra of the signal at all channel locations for the two conditions, eyes open and eyes closed. Right: scalp plot of the signal power at $10 \mathrm{~Hz}$ for the two conditions (eyes open and closed). The actual noise of the artificial data varied linearly in time between both conditions, depending of the task of the $\mathrm{BCl}$ user.

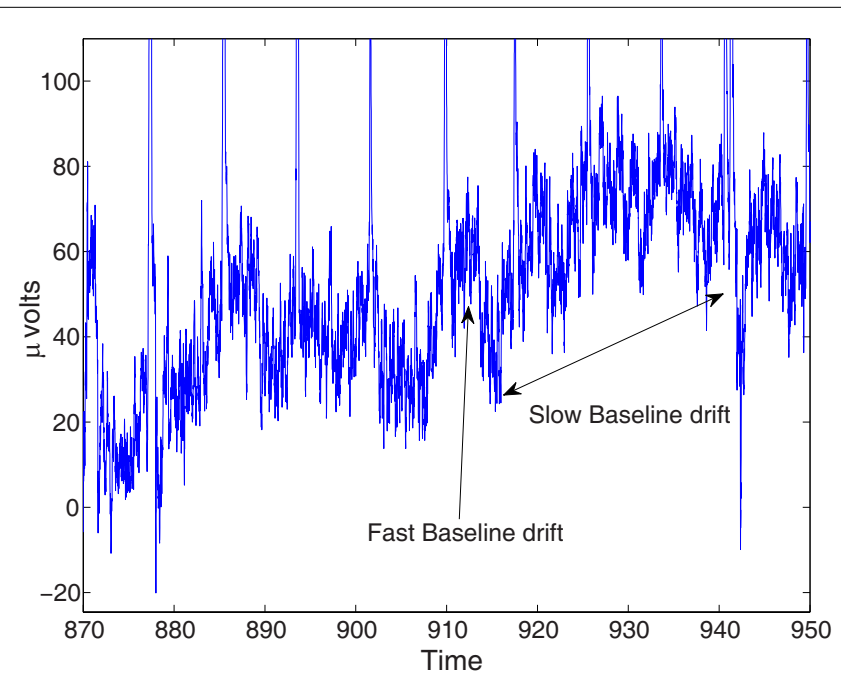

FIGURE 9 | (Data Set 1 - artificial). Example of fast and slow baseline drifts that are observable in unfiltered $\mathrm{BCl}$ competition IV data. The figure depicts the time course of the amplitude of the EEG in one channel.

with conductivity ratios 1:0.02:1. The Maxwell equations were solved using an analytic expansion of the EEG lead field (Nolte and Dassios, 2005).

Both left and right rhythmic activity was present in all conditions. However, during left hand movements the right side rhythmic activity was reduced by at least $50 \%$ (it changed slightly for each data set) and vice versa.

\subsubsection{Harmonic oscillations in the beta band}

A harmonic component of the subject-specific $\mu$ rhythm can often be observed in the $\beta$ band (Huber et al., 1971; Pfurtscheller, 1981; Pfurtscheller et al., 1996; Pfurtscheller and Lopes da Silva, 1999; Carlqvist et al., 2004; Nikulin et al., 2007). In our data sets we have included such a harmonic component with different levels of amplitude in relation to the $\mu$ rhythm, varying from 15 to $1 \%$ (see Figure 12).

\subsubsection{Asymmetry in the amplitude of the rhythms}

Typically, one can observe some asymmetry in the strength of the desynchronization in each of the hemispheres (McFarland et al., 2000; Mazaheri and Jensen, 2008; Nikulin et al., 2010). In a pair of data sets and in order to create a more realistic EEG we added this asymmetry in the rhythms that we generated.

\subsubsection{Generation of artifacts}

Both for eye blinks and eye movements we assumed the generators to be current dipoles placed within the eyes. The dipoles in the left and right eye were activated simultaneously in a randomly chosen superposition of a vertical and horizontal direction. The potentials due to vertical dipoles were on average 10 times stronger than the ones from horizontal direction. The topographies of vertical and horizontal dipoles are shown in the upper panels of Figure 13.

While the spatial patterns were (on average) identical for eye movement and eye blinks, the time courses were chosen differently. Time courses of eye movements were modeled as constants with continuous on- and offsets as shown in the lower left panel of Figure 13. The duration of the constant was set randomly between 0 and $2 \mathrm{~s}$.

The time course of eye blinks was chosen as

$x(t)=(t+\xi) \exp \left(-\frac{t^{2}}{2 \sigma_{t}^{2}}\right)$

with a width set to $\sigma_{t}=31 \mathrm{~ms}$ according to real eye blinks and with $x i$ being a Gaussian distributed random variable with standard deviation equal to $20 \mathrm{~ms}$. An example time course is given in the lower right panel of Figure 13.

\subsubsection{Combining the ingredients}

For each data set, the final EEG was generated by the linear combination of each element (background noise, baseline drifts, ERD, and eye artifacts). The background noise was a superposition of the cross-spectra in the conditions eyes open and eyes closed. The amount of each type of cross-spectrum depended on the "environmental" conditions in which the virtual user was supposed to be immersed: visual load (large amount of eyes open condition and small amount of eyes closed condition), auditive load (small amount of eyes open condition and larger amount of eyes closed condition).

The ERD frequency was randomly chosen between 10 and $12 \mathrm{~Hz}$ for each user and a harmonic in the beta band (by doubling the $\mu$ rhythm frequency) was added as well. The position of the dipoles generating the oscillatory activity could vary slightly and randomly for each user. As already described, we also allowed asymmetry of the $\mu$ rhythm amplitude in each hemisphere. 

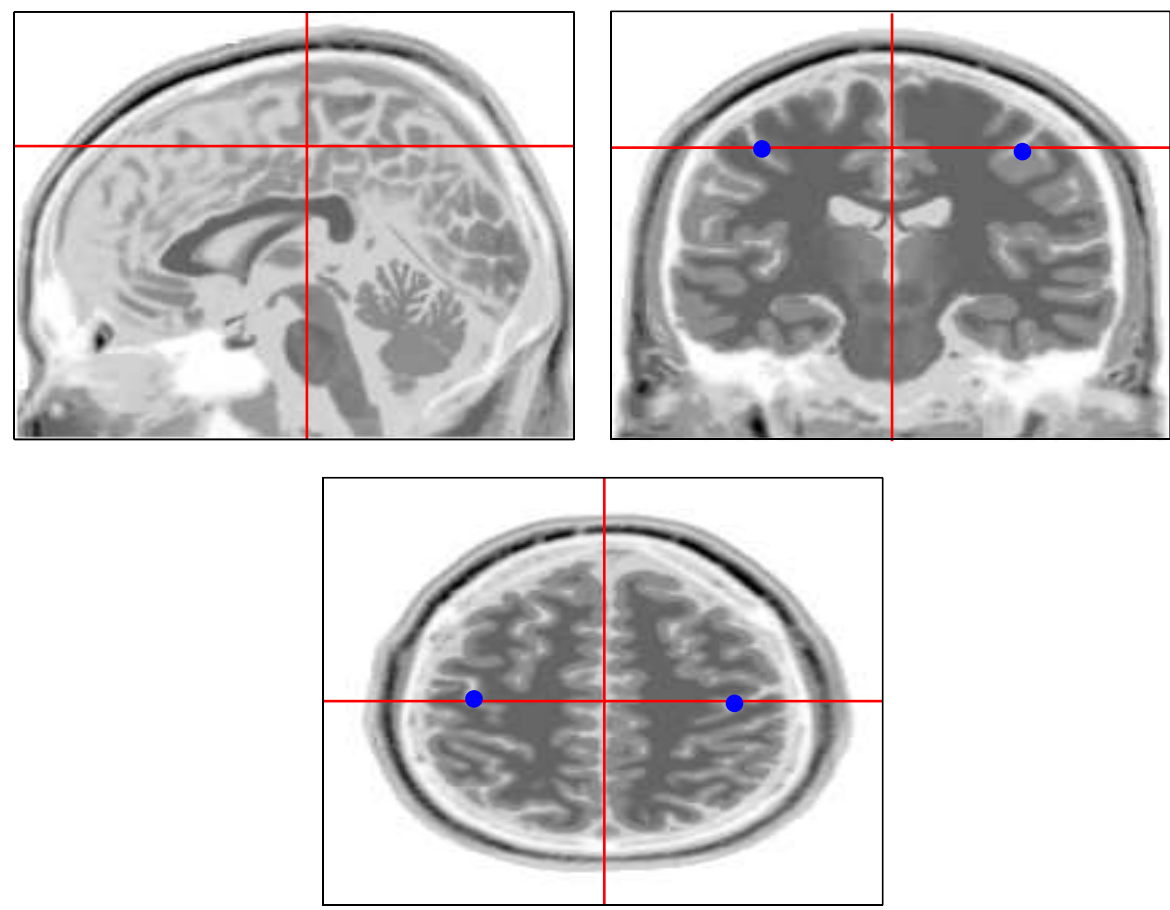

FIGURE 10 | (Data Set 1 - artificial). Location and direction of selected dipoles in the head.

Then the eye movements and eye blinks were as well superimposed to the signal. One random time course was generated for each of the data sets. Finally, the baseline drifts were added to the total.

For the calculation, each element (ERD, background noise, etc.) was normalized by its trace and the coefficient multiplying each of them was manually selected, by computing the expected performance using baseline methods (frequency band and time interval subject-selected, then CSP computed using training data and applied to test data). For more information please refer to (Blankertz et al., 2008b).

\subsection{Challenge}

As it was not revealed which of the data sets were real and which were artificially generated. The challenge and evaluation criterion was identical, see Section 3.4.

\subsection{OUTCOME IN BRIEF}

A comparison of the similarity of real and synthetic data was performed based on the result ranking (available via http://bbci.de/competition/iv/results/). First, we analyzed the position of the participants in the ranking. We calculated the correlation coefficient of the participants' positions in both of the data sets and obtained a result of 0.89 , meaning that the position of a participant in both rankings was highly correlated: a good rank in the real data analysis yielded a good rank in the artificial data analysis and vice versa. Also, we analyzed the performance of the participants in the same way. We obtained a correlation coefficient of 0.93 , meaning that the performance of a participant in both data sets was very similar. The linear fitting had a slight

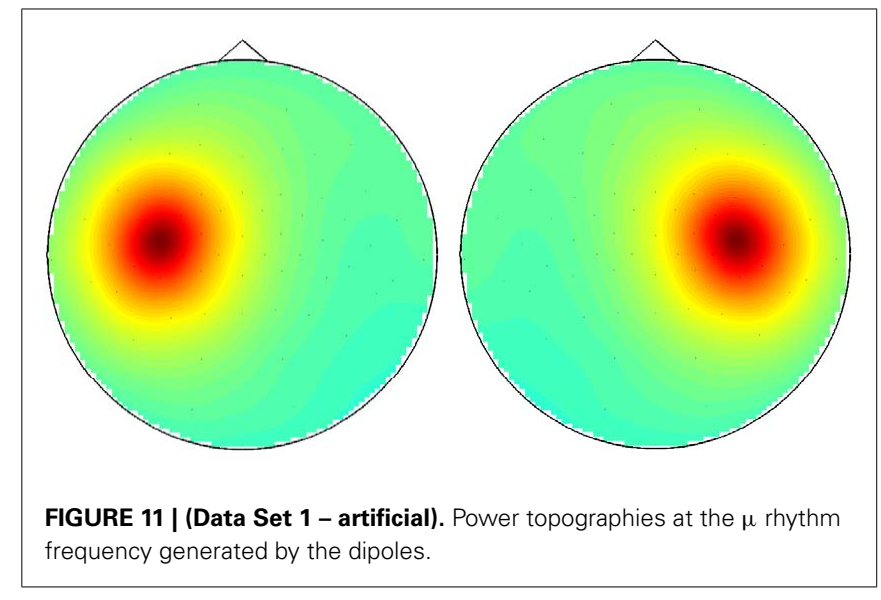

positive bias (0.02), which shows that the performance measurement (mean squared error) was slightly higher for the synthetic EEG (these data sets were a bit noisier than the real ones).

Summarizing, those algorithms doing well in the real data sets also performed higher in the artificial data and vice versa.

\subsection{DISCUSSION}

In this section we gave a description of the generation of synthetic EEG. We have described all its components, documented our decisions, and detailed the calculation of each element.

We emphasize that more sophisticated EEG forward models would include CSF as a fourth layer and that new research indicates that the chosen conductivity ratio (1:50) might be too high. While the simulation could be improved, almost all BCI methods work 
entirely in sensor space and the exact details of the topographies will hardly affect the results of the BCI task.

We have analyzed the results of the BCI competition IV and shown the high correlation between the ranking and the performance measure in the real and artificial EEG in Figure 14. In this specific context, the creation of synthetic EEG data sets has proven to be useful.

Artificially generated EEG can be generated in large amounts. Using it not only avoids performing real recordings, it can also be fine-tuned, e.g., to contain a controlled amount of certain artifacts. Both characteristics are beneficial for an initial performance evaluation of new algorithmic methods.

Although this was our first try to generate artificial data and the methods can be further developed, we have shown a way to create

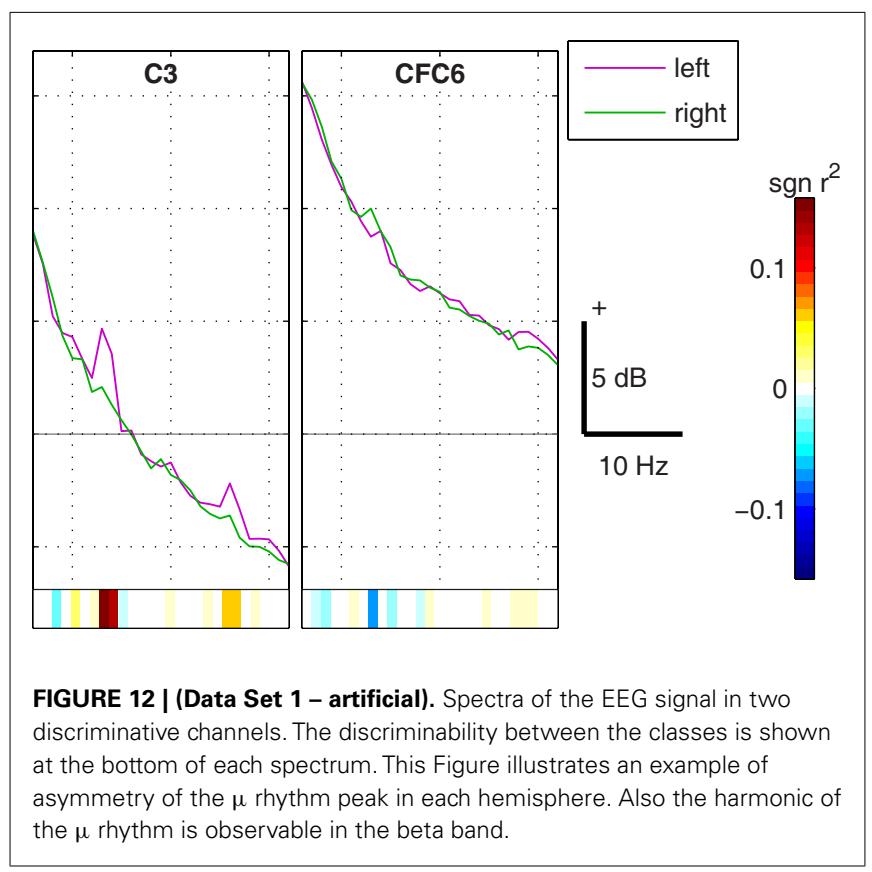

data under controlled conditions, in order to test new methods before performing actual experiments and this way boosting the probability of success of new analysis methods in neuroscience.

In the future we will work on the improvement of the artifact generation methods and develop an automatic way to combine all the components of our synthetic electroencephalogram. Additionally, more tests should be done with the artificially generated data, to assure that the correlation between real and synthetic EEG is as high as shown in this report.

\section{DATA SET 2A}

Data set 2a Continuous Multi-class Motor Imagery is provided by C. Brunner, R. Leeb, G. R. Müller-Putz, G. Pfurtscheller, and

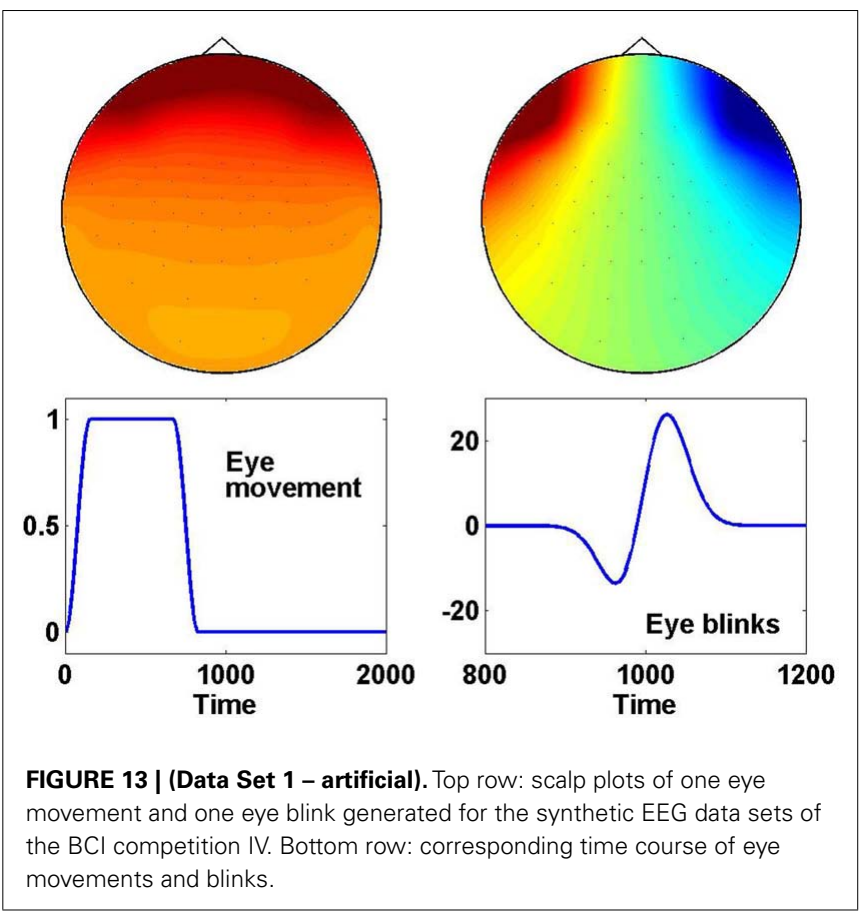

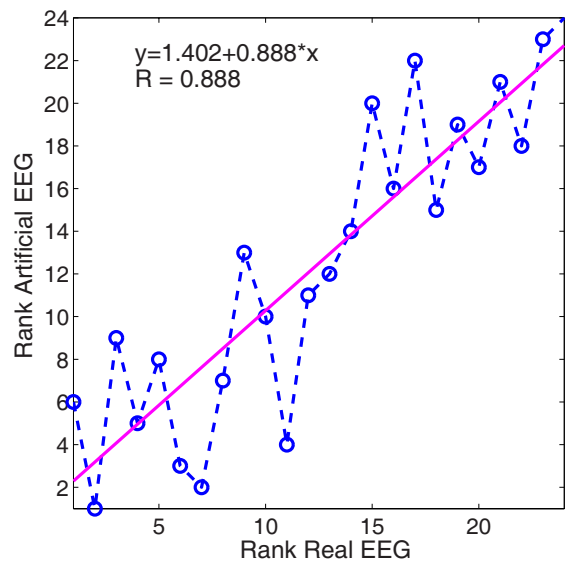

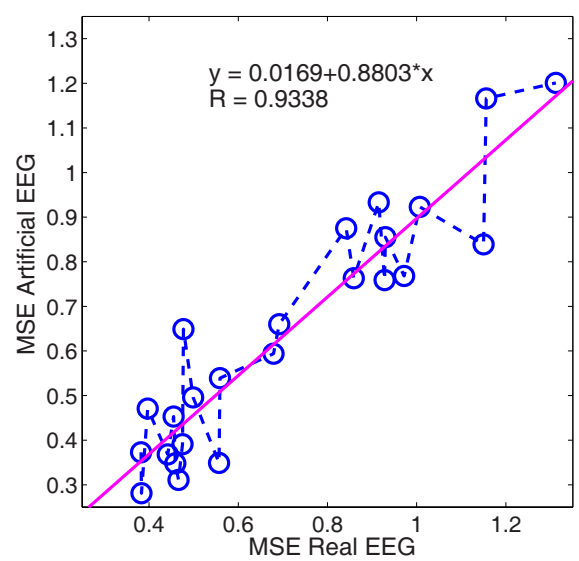

FIGURE 14 | (Data Set 1 - artificial). Linear regression of the rank position (left) and performance of the method (right). The x-axis corresponds to the results submitted for the real EEG data sets, whereas $y$-axis corresponds to those of the synthetic EEG. 
A. Schlögl from Graz (Austria). It can be freely assessed via http://www.bbci.de/competition/iv/ with the only restriction that the present article is referenced upon any publication of results.

\subsection{MOTIVATION}

This data set challenges the session-to-session transfer of a three class motor imagery task. Compared to other synchronous motor imagery data sets, a continuous estimation of motor imagery class labels is required. This represents a realistic setting for an online control of a continuous output parameter.

\subsection{MATERIALS AND SUBJECTS}

This data set comprises electroencephalographic (EEG) data from 9 subjects.

\subsubsection{Experimental paradigm}

The cue-based BCI paradigm consisted of four different motor imagery tasks, namely the imagination of movement of the left hand (class 1), right hand (class 2), both feet (class 3), and tongue (class 4). Two sessions on different days were recorded for each subject. Each session is comprised of 6 runs separated by short breaks. One run consists of 48 trials ( 12 for each of the four possible classes), yielding a total of 288 trials per session.

\subsubsection{Protocol}

At the beginning of each session, we recorded approximately 5 min of EEG data to estimate the EOG influence. This recording was divided into 3 blocks: (1) 2 min with eyes open (looking at a fixation cross on the screen), (2) 1 min with eyes closed, and (3) 1 min with eye movements. The timing scheme of one session is illustrated in Figure 15. Note that due to technical problems, the EOG block is shorter for subject A04T and contains only the eye movement condition (see Table A1 in Appendix for a list of all subjects).

All subjects were sitting in a comfortable armchair in front of a computer screen. At the beginning of a trial ( $t=0 \mathrm{~s})$, a fixation cross appeared on the black screen. In addition, a short acoustic warning tone was presented. After $2 \mathrm{~s}(t=2 \mathrm{~s})$, a cue in the form of an arrow pointing either to the left, right, down, or up (corresponding to one of the four classes left hand, right hand, foot, or tongue) appeared and stayed on the screen for $1.25 \mathrm{~s}$. This prompted the subjects to perform the desired motor imagery task. No feedback was provided. The subjects were instructed to carry out the motor imagery task until the fixation cross disappeared from the screen at $t=6 \mathrm{~s}$. A short break with a black screen followed. The paradigm is illustrated in Figure $\mathbf{1 6 .}$

\subsection{DATA FORMAT}

Twenty-two Ag/AgCl electrodes (with inter-electrode distances of $3.5 \mathrm{~cm}$ ) were used to record the EEG; the montage is shown in

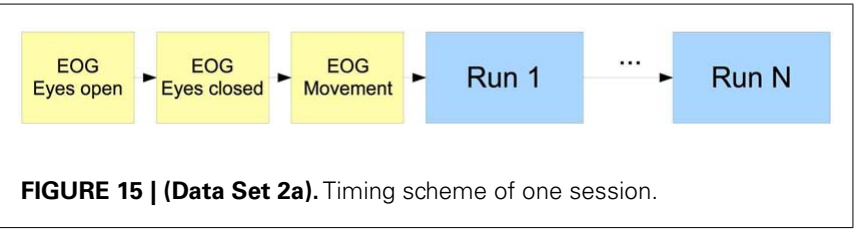

Figure 17, left. All signals were recorded monopolarly with the left mastoid serving as reference and the right mastoid as ground. The signals were sampled with $250 \mathrm{~Hz}$ and bandpass filtered between 0.5 and $100 \mathrm{~Hz}$. The sensitivity of the amplifier was set to $100 \mu \mathrm{V}$. An additional $50 \mathrm{~Hz}$ notch filter was enabled to suppress line noise.

In addition to the 22 EEG channels, 3 monopolar EOG channels were recorded and also sampled with $250 \mathrm{~Hz}$ (see Figure 17, right). They were bandpass filtered between 0.5 and $100 \mathrm{~Hz}$ (with the 50$\mathrm{Hz}$ notch filter enabled), and the sensitivity of the amplifier was set to $1 \mathrm{mV}$. The EOG channels are provided for the subsequent application of artifact processing methods (Fatourechi et al., 2007) and must not be used for classification.

A visual inspection of all data sets was carried out by an expert and trials containing artifacts were marked. Eight out of the total of nine data sets were analyzed in Naeem et al. (2006) and Brunner et al. $(2007,2011)$.

All data sets are stored in the general data format for biomedical signals (GDF), one file per subject and session. However, only one session contains the class labels for all trials, whereas the other sessions are used to test the classifier and hence to evaluate the performance. For details on the data set, the GDF files contained, markers and functions provided for loading and evaluation, please see Section A.1 in Appendix.

\subsection{CHALLENGE}

Participants were asked to provide a continuous classification output for each sample in the form of class labels (1-4), including labeled trials and trials marked as artifact. A confusion matrix was then built from all artifact-free trials for each time point. From these confusion matrices, the time course of the accuracy as well as the kappa coefficient was obtained (Schlögl et al., 2007b). The chance level was at $\kappa=0$. The algorithm used for this evaluation was provided in BioSig. The algorithm achieving the largest kappa value was declared the winner.

Due to the fact that the test data sets were not distributed until the end of the competition, software had to be submitted. It had to be capable to process EEG data files of the same format as used for all training sets ${ }^{2}$ ) and produce the aforementioned class label vector.

Since three EOG channels were provided, the software was required to remove EOG artifacts before the subsequent data processing using artifact removal techniques such as high pass filtering

${ }^{2}$ One test data set is distributed from the beginning of the competition to enable participants to test their program and to ensure that it produces the desired output.

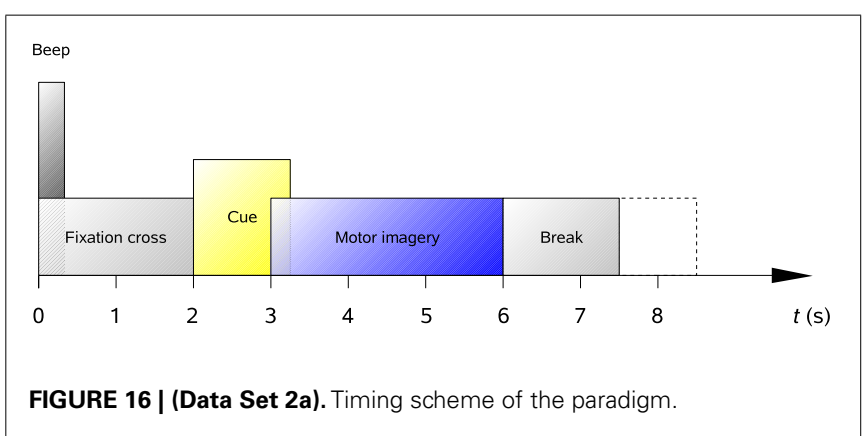




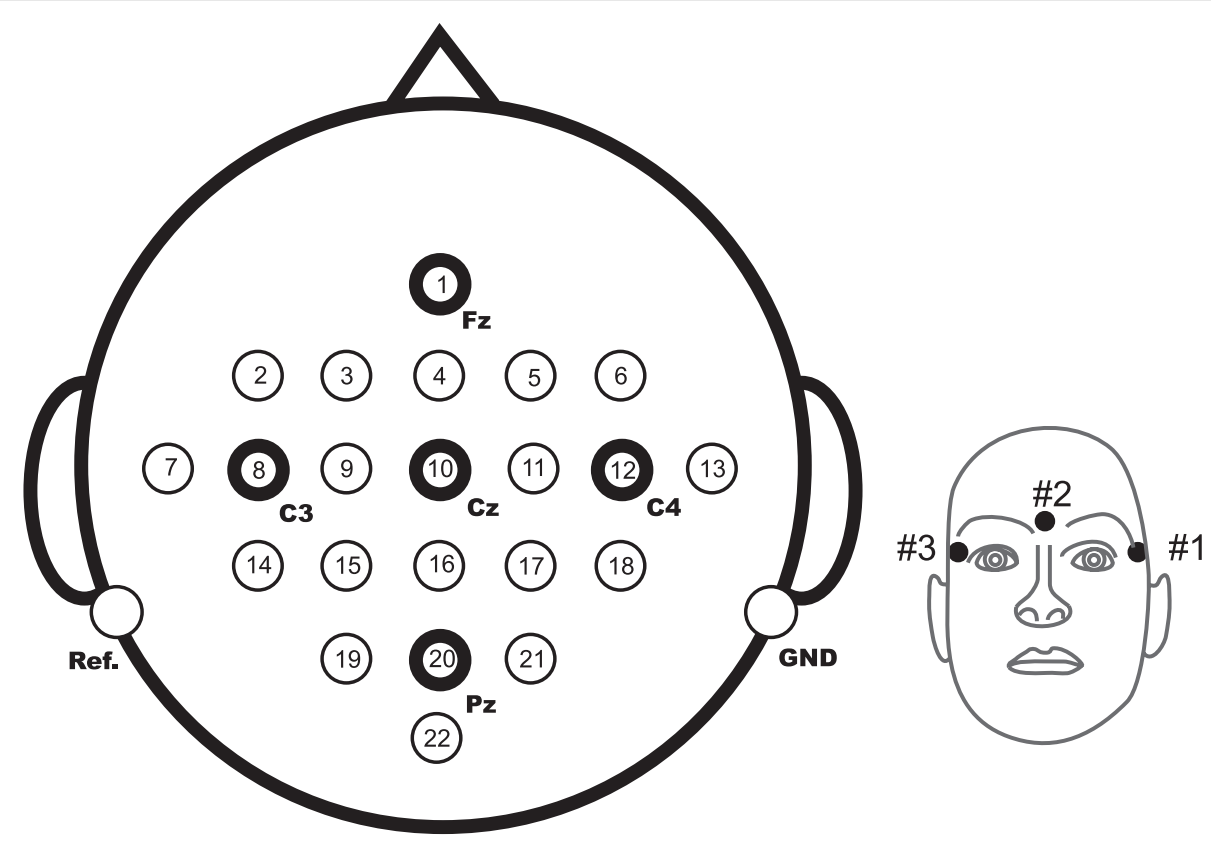

FIGURE 17 | (Data Set 2a). Left: electrode montage corresponding to the international 10-20 system. Right: electrode montage of the three monopolar EOG channels.

or linear regression (Schlögl et al., 2007a). The use of other correction methods was possible, but it was requested that artifacts had no influence on the classification results.

All algorithms had to be causal, meaning that the classification output at time $k$ was allowed only to depend on the current and past samples $x_{k}, x_{k-1}, \ldots, x_{0}$. In order to check whether the causality criterion and the artifact processing requirements were fulfilled, all submissions had to be open source, including all additional libraries, compilers, programming languages, and so on (for example, Octave/FreeMat, $\mathrm{C}++$, Python, etc.). Note that submissions could also be written in the closed-source development environment MATLAB as long as the code was executable in Octave. Similarly, $\mathrm{C}++$ programs could be written and compiled with a Microsoft or Intel compiler, but the code had to compile also with $\mathrm{g}++$.

\subsection{OUTCOME IN BRIEF}

There were five submissions for this data set (see Table 3). All of them used CSP features. The winning algorithm was submitted by K. K. Ang, Z. Y. Chin, C. Wang, C. Guan, H. Zhang, K. S. Phua, B. Hamadicharef, and K. P. Tee from the Institute for Infocomm Research, Agency for Science, Technology and Research Singapore. Details of their approach are described in Ang et al. (2012). The performance measure kappa was 0.57 averaged over all nine subjects. The other four submissions attained kappa values of 0.52 , $0.31,0.30$, and 0.29 and thus were well above chance level of $\kappa=0$. The winning algorithm performed best in seven out of nine subjects; in two subjects, the algorithm that overall ranked second best reached even slightly higher kappa values.

The winning algorithm requires MATLAB, but also runs on Octave. It uses the BioSig toolbox to load the data. The algorithm
Table 3 | (Data Set 2a). Contributions with final result (kappa).

\begin{tabular}{|c|c|c|}
\hline Contributor & Kappa & Lab \\
\hline K. K. Ang & 0.57 & $\begin{array}{l}\text { Institute for Infocomm Research, Agency for } \\
\text { Science, Technology and Research Singapore }\end{array}$ \\
\hline L. Guangquan & 0.52 & $\begin{array}{l}\text { School of Mechanical Engineering, Shanghai } \\
\text { Jiao Tong University, China }\end{array}$ \\
\hline W. Song & 0.31 & $\begin{array}{l}\text { College of Inf. Science and Techn., Beijing } \\
\text { Normal University, China and National Key } \\
\text { Lab. or Cog. Neurosc. and Learning, Beijing } \\
\text { Normal Univ., China }\end{array}$ \\
\hline D. Coyle & 0.30 & $\begin{array}{l}\text { Intelligent Systems Research Centre, School } \\
\text { of Computing and Intell. Systems, Faculty of } \\
\text { Computing and Eng., Magee Campus, } \\
\text { University of Ulster, UK }\end{array}$ \\
\hline J.Wu & 0.29 & $\begin{array}{l}\text { National Key Lab. for Cogn. Neurosc. and } \\
\text { Learning, Beijing Normal Univ., China and } \\
\text { College of Inf. Science and Techn., Beijing } \\
\text { Normal University, China }\end{array}$ \\
\hline
\end{tabular}

is based on the filter bank common spatial pattern (FBCSP) variant (Ang et al., 2008). It was extended to the multi-class case with one-versus-the-rest classifiers. First, artifacts were removed by bandpass filters. Each classifier selected discriminative CSP features using the Mutual Information Best Individual Features (MIBIF4) algorithm (Ang and Quek, 2006) before Naive Bayes Parzen Window classifiers (Ang and Quek, 2006) were used. The classifier with the highest probability yielded the overall classification result. Due to the computationally intensive algorithms, classification was performed every ten samples (in combination 
with a zero-order hold for the samples in between). As the algorithm used $2 \mathrm{~s}$ of EEG data, the classification output was delayed by $2 \mathrm{~s}$.

\subsection{DISCUSSION}

All five submissions yielded results well above chance level. As a side note, four contributions were submitted by Asian-Pacific groups. As already mentioned above, all contributions used CSP features.

There were two major challenges in this data set. First, the contamination with eye movement artifacts could affect classification accuracy; therefore we provided additional EOG channels. Second, the classifiers trained on the training sessions should generalize on unseen data recorded on a different day. The winning algorithm addressed the first issue with a simple bandpass filter. Obviously, the method is stable because it yielded good results on the test data. However, the classification output is delayed by $2 \mathrm{~s}$, which could be a problem in online BCIs that incorporate real-time feedback.

\section{DATA SET 2B}

Data set 2b Session-to-Session Transfer of a Motor Imagery BCI under Presence of Eye Artifacts is provided by R. Leeb, C. Brunner, G. R. Müller-Putz, and G. Pfurtscheller from Graz (Austria). It can be freely assessed via http://www.bbci.de/competition/iv/ with the only restriction that the present article is referenced upon any publication of results.

\subsection{MOTIVATION}

This data set focuses on the classification of electroencephalogram (EEG) signals affected by eye movement artifacts. Furthermore the session-to-session transfer of the algorithms has to be taken in consideration, because all training and test data sets are recorded on five different days.

The data set $2 \mathrm{~b}$ contains the electroencephalogram (EEG) and electrooculogram (EOG) activity of nine subjects. Technically speaking, each data set consists of single-trials of spontaneous brain activity during motor imagery, one part labeled (training data) and another part unlabeled (test data), and a performance measure. The goal is to infer labels (or their probabilities) for the test data sets from training data that maximize the performance measure for the true (but to the competitors unknown) labels of the test data (this information is now, after the competition, available as well).

\subsection{MATERIALS AND SUBJECTS}

This data set consists of EEG data from 9 subjects of a study published in Leeb et al. (2007). The subjects were right-handed, had normal or corrected-to-normal vision and were paid for participating in the experiments. All volunteers were sitting in an armchair, watching a flat screen monitor placed approximately $1 \mathrm{~m}$ away at eye level. For each subject 5 sessions are provided, whereby the first two sessions contain training data without feedback (screening), and the last three sessions were recorded with feedback.

\subsubsection{Experimental paradigm}

Each session consists of several runs, illustrated in Figure 18. At the beginning of each session, a recording of approximately $5 \mathrm{~min}$

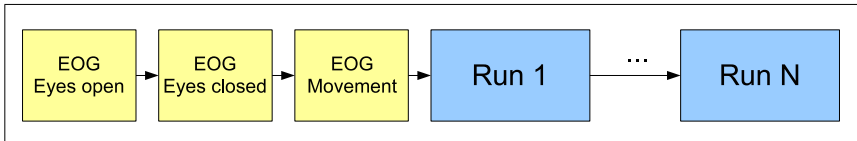

FIGURE 18 | (Data Set 2b). Timing scheme of one session (for screening and feedback sessions).

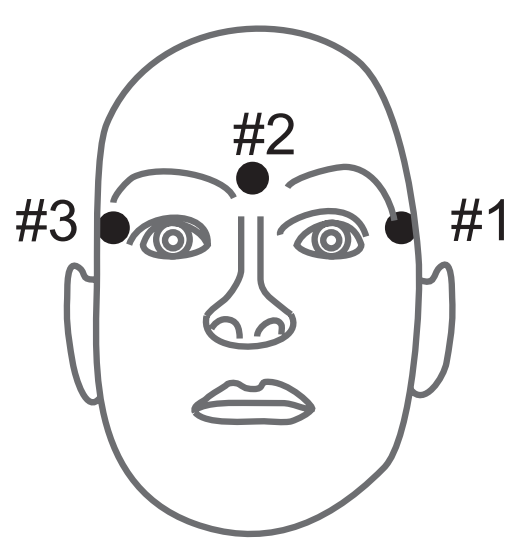

FIGURE 19 | (Data Set 2b). Electrode montage of the three monopolar EOG channels.

was performed to estimate the EOG influence. The recording was divided into 3 blocks: (1) 2 min with eyes open (looking at a fixation cross on the screen), (2) 1 min with eyes closed, and (3) 1 min with eye movements. The artifact block was divided into four sections ( $15 \mathrm{~s}$ artifacts with $5 \mathrm{~s}$ resting in between) and the subjects were instructed with a text on the monitor to perform either eye blinking, rolling, up-down, or left-right movements. At the beginning and at the end of each task a low and high warning tone were presented, respectively. Note that due to technical problems no EOG block is available in session B0102T and B0504E (see Table A3 in Appendix for a list of all subjects).

\subsubsection{Protocol}

Three bipolar recordings $(\mathrm{C} 3, \mathrm{Cz}$, and $\mathrm{C} 4)$ were recorded with a sampling frequency of $250 \mathrm{~Hz}$. The recordings had a dynamic range of $\pm 100 \mu \mathrm{V}$ for the screening and $\pm 50 \mu \mathrm{V}$ for the feedback sessions. They were bandpass filtered between 0.5 and $100 \mathrm{~Hz}$, and a notch filter at $50 \mathrm{~Hz}$ was enabled. The placement of the three bipolar recordings (large or small distances, more anterior or posterior) were slightly different for each subject (for more details see Leeb et al., 2007). The electrode position Fz served as EEG ground.

In addition to the EEG channels, the electrooculogram (EOG) was recorded with three monopolar electrodes (see Figure 19, left mastoid serving as reference) using the same amplifier settings, but with a dynamic range of $\pm 1 \mathrm{mV}$. The EOG channels are provided for the subsequent application of artifact processing methods (Fatourechi et al., 2007) and must not be used for classification.

The cue-based screening paradigm (see Figure 20A) consisted of two classes, namely the motor imagery (MI) of left hand (class 1) and right hand (class 2). Each subject participated in two screening 


\section{A Screening}
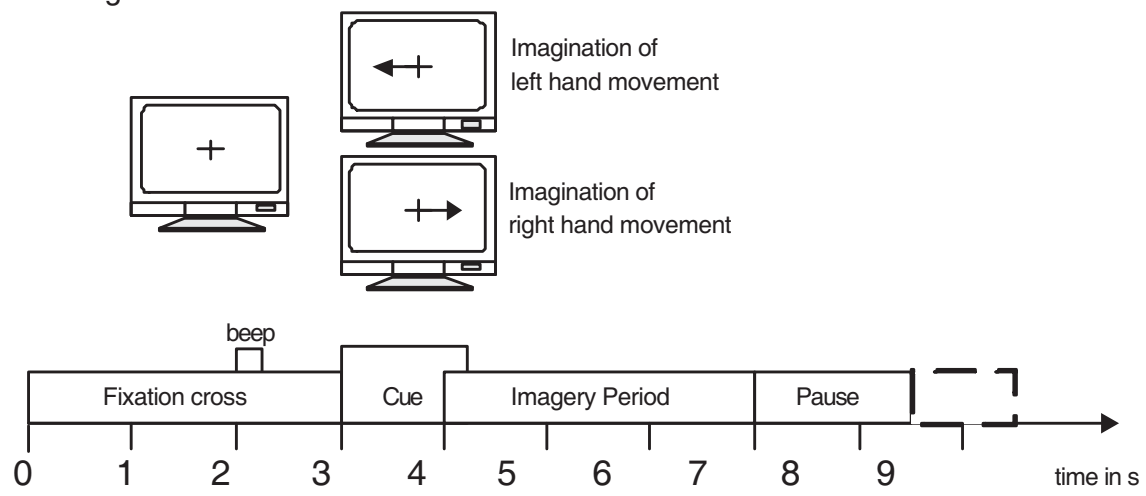

B Smiley Feedback

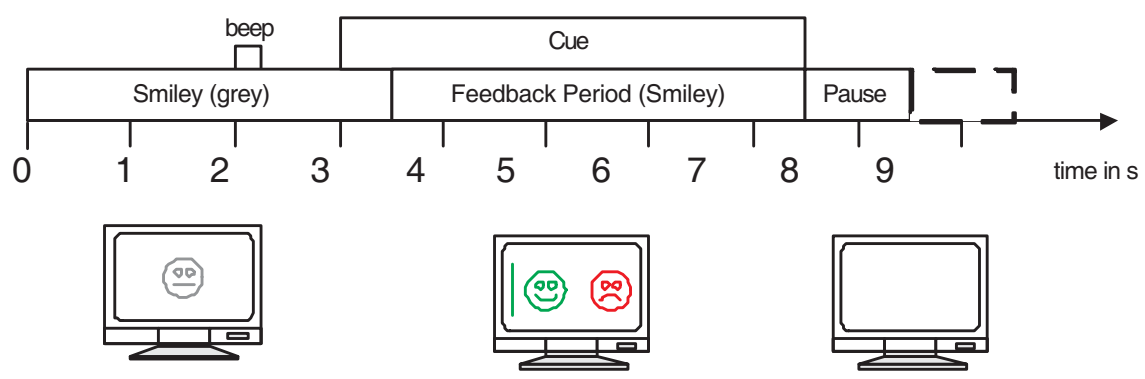

FIGURE 20 | (Data Set 2b). Timing scheme of the paradigm. (A) The first two sessions (01T, 02T) contain training data without feedback, and (B) the last three sessions (03T, 04E, 05E) with smiley feedback.

sessions without feedback recorded on two different days within 2 weeks. Each session consisted of six runs with ten trials each and two classes of imagery. This resulted in 20 trials per run and 120 trials per session. Data of 120 repetitions of each MI class were available for each person in total. Prior to the first motor imagery training the subject executed and imagined different movements for each body part and selected the one which they could imagine best (e. g., squeezing a ball or pulling a brake).

Each trial started with a fixation cross and an additional short acoustic warning tone $(1 \mathrm{kHz}, 70 \mathrm{~ms})$. Some seconds later a visual cue (an arrow pointing either to the left or right, according to the requested class) was presented for $1.25 \mathrm{~s}$. Afterward the subjects had to imagine the corresponding hand movement over a period of $4 \mathrm{~s}$. Each trial was followed by a short break of at least $1.5 \mathrm{~s}$. A randomized time of up to $1 \mathrm{~s}$ was added to the break to avoid adaptation.

For the three online feedback sessions four runs with smiley feedback were recorded (see Figure 20B), whereby each run consisted of twenty trials for each type of motor imagery. At the beginning of each trial (second 0 ) the feedback (a gray smiley) was centered on the screen. At second 2, a short warning beep $(1 \mathrm{kHz}$, $70 \mathrm{~ms}$ ) was given. The cue was presented from seconds 3 to 7.5. Depending on the cue, the subjects were required to move the smiley toward the left or right side by imagining left or right hand movements, respectively. During the feedback period the smiley changed to green when moved in the correct direction, otherwise it became red. The distance of the smiley from the origin was set according to the integrated classification output over the past $2 \mathrm{~s}$ (more details see Leeb et al., 2007). Furthermore, the classifier output was also mapped to the curvature of the mouth causing the smiley to be happy (corners of the mouth upwards) or sad (corners of the mouth downward). At second 7.5 the screen went blank and a random interval between 1.0 and $2.0 \mathrm{~s}$ was added to the trial. The subject was instructed to keep the smiley on the correct side for as long as possible and therefore to perform the MI as long as possible.

\subsection{DATA FORMAT}

All data sets are stored in the general data format for biomedical signals (GDF), one file per subject and session. However, only the first three sessions contain the class labels for all trials, whereas the remaining two sessions are used to test the classifier and hence to evaluate the performance. For details on the data set, the GDF files contained, markers and functions provided for loading and evaluation, please see Section A.2 in Appendix.

\subsection{ChAllenge}

Participants were asked to provide a continuous classification output for each sample in the form of class labels $(1,2)$, including labeled trials and trials marked as artifacts. A confusion matrix was then built based on artifact-free trials only and for each time point. From these confusion matrices, the time course of the accuracy as well as the kappa coefficient was obtained (Schlögl et al., 2007b), which had a chance level of $\kappa=0$. The algorithm used 
for this evaluation was provided in BioSig. The winner was the algorithm with the largest kappa value.

Due to the fact that the evaluation data sets were not distributed until the end of the competition, software had to be submitted. It had to be capable to process EEG data files of the same format as used for all training sets ${ }^{3}$ ) and produce the aforementioned class label vector.

Since three EOG channels were provided, the software was required to remove EOG artifacts before the subsequent data processing using artifact removal techniques such as high pass filtering or linear regression (Schlögl et al., 2007a). The use of other correction methods was possible, but it was requested that artifacts had no influence on the classification results.

All algorithms were required to be causal, meaning that the classification output at time $k$ may only depend on the current and past samples $x_{k}, x_{k-1}, \ldots, x_{0}$. In order to check whether the causality criterion and the artifact processing requirements were fulfilled, all submissions had to be submitted as open source, including all additional libraries, compilers, programming languages, and so on (for example, Octave/FreeMat, $\mathrm{C}++$, Python, etc.). Note that submissions could also be written in the closed-source development environment MATLAB as long as the code was executable in Octave. Similarly, $\mathrm{C}++$ programs could be written and compiled with a Microsoft or Intel compiler, but the code had to compile also with $g++$.

\subsection{SUBMISSIONS AND ALGORITHMS}

Six groups submitted their participation for this data set. The given list is in winning order and this ID will be used further on:

ID-1: Zheng Yang Chin, Kai Keng Ang, Chuanchu Wang, Cuntai Guan, Haihong Zhang, Kok Soon Phua, Brahim Hamadicharef, and Keng Peng Tee from the Institute for Infocomm Research, Agency for Science, Technology, and Research in Singapore.

ID-2: Huang Gan, Liu Guangquan, and Zhu Xiangyang from the School of Mechanical Engineering, Shanghai Jiao Tong University in China.

ID-3: Damien Coyle, Abdul Satti, and Martin McGinnity from the Intelligent Systems Research Centre, School of Computing and Intelligent Systems, Faculty of Computing and Engineering, Magee Campus, University of Ulster in the United Kingdom.

ID-4: Shaun Lodder and Johan du Preez from the E\&E Engineering, University of Stellenbosch in South Africa.

ID-5: Jaime Fernando Delgado Saa from the Robótica y Sistemas Inteligentes, Universidad del Norte in Colombia.

ID-6: Yang Ping, Xu Lei, and Yao Dezhong from the PerceptionMotor Interaction Lab, School of Life Science and Technology, University of Electronic Science and Technology in China.

For each method the applied preprocessing, feature extraction, and classification steps are briefly given.

Methods participant ID-1: They authors removed the EOG with a bandpass filter and extracted their features via a Filter Bank ${ }^{3}$ One test data set is distributed from the beginning of the competition to enable
participants to test their program and to ensure that it produces the desired output.
CSP (FBCSP) using mutual information rough set reduction (MIRSR). Classification of selected CSP features was performed using the Naïve Bayes Parzen Window classifier. A more detailed explanation of the winning algorithm is given in a separate paper (Ang et al., 2012).

Methods participant ID-2: The EEG was bandpass filtered in different frequency bands and the EOG artifacts were removed afterward. Common spatial subspace decomposition (CSSD) were extracted from the preprocessed signals with optimized window sizes and a LDA discriminate function was made for each time point.

Methods participant ID-3: CSP on spectrally filtered neural time series prediction preprocessing (NTSPP) signals was applied to all signals all subjects using the self-organizing fuzzy neural network (SOFNN). Furthermore the log variance of each filtered channel was calculated with a 1-s sliding window. The best classifier among 3 variants of LDA and 2 variants of SVM was chosen for each subject individually.

Methods participant ID-4: Wavelet packet transform was applied only on electrodes $\mathrm{C} 3$ and $\mathrm{C} 4$ ( $\mathrm{Cz}$ was ignored). Selected frequency bands were extracted and concatenated to form a multidimensional vector and classified with LDA.

Methods participant ID-5: EOG was removed with linear regression and the signals high pass filtered with $4 \mathrm{~Hz}$. The algorithm used spectral features in the mu and beta bands (from electrode C3 and C4) as inputs for a neural network classifier.

Methods participant ID-6: EOG was removed with linear regression. Band-power features in 75 frequency bands for each channel were extracted and selected with recursive feature elimination (RFE). The remaining 6 features were classified with a Bayesian LDA.

\subsection{RESULTS}

In total six submissions were received and most were of high quality. As defined above in section evaluation the kappa value was chosen as the performance measure. Remember, the expected kappa value, if classification is made by chance, is 0 . In Table 4 the first column shows the average kappa across all subjects, columns $2-10$ show the results for the individual subjects. Four submissions achieved a mean kappa of more than 0.4 on the test set. Furthermore the two best approaches (ID-1 and ID-2; Ang et al., 2012) achieved nearly similar results (mean of 0.60 and 0.58 ). Actually approach ID-2 could achieve the best single subject performances for 4 subjects and ID-1 "just" for 3 subjects, but was always very close to the best ones on a single subject level. Only subject 2 caused troubles to these algorithms. Interestingly is that approach ID-4 achieved incredible good results here compared to the other approaches. Generally the data from subject 8 and subject 4 could be identified best, whereby subjects 3,2 , and 1 were challenging. These findings are consistent over all approaches, if the standard deviation over the approaches is taken into consideration.

\subsection{DISCUSSION}

Two major challenges had to be addressed in this data set. The first one was the influence of eye movement artifacts on the EEG and the second one the generalization of the selected features to be successful on the session-to-session transfer. Like in real conditions 
Table 4 | (Data Set 2b). Detailed results from the BCl competition IV.

\begin{tabular}{|c|c|c|c|c|c|c|c|c|c|c|}
\hline \multirow[t]{2}{*}{ Part. ID } & \multirow[t]{2}{*}{ Mean } & \multicolumn{9}{|c|}{ Subject } \\
\hline & & 1 & 2 & 3 & 4 & 5 & 6 & 7 & 8 & 9 \\
\hline ID-1 & 0.60 & 0.40 & 0.21 & 0.22 & 0.95 & 0.86 & 0.61 & 0.56 & 0.85 & 0.74 \\
\hline ID-2 & 0.58 & 0.43 & 0.21 & 0.14 & 0.94 & 0.71 & 0.62 & 0.61 & 0.84 & 0.78 \\
\hline ID-3 & 0.46 & 0.19 & 0.12 & 0.12 & 0.77 & 0.57 & 0.49 & 0.38 & 0.85 & 0.61 \\
\hline ID-4 & 0.43 & 0.23 & 0.31 & 0.07 & 0.91 & 0.24 & 0.43 & 0.41 & 0.74 & 0.53 \\
\hline ID-5 & 0.37 & 0.20 & 0.16 & 0.16 & 0.73 & 0.21 & 0.21 & 0.39 & 0.86 & 0.44 \\
\hline ID-6 & 0.25 & 0.02 & 0.09 & 0.07 & 0.43 & 0.25 & 0.00 & 0.14 & 0.76 & 0.47 \\
\hline
\end{tabular}

Kappa values for each subject and the mean kappa for all participating groups.

the data were from different sessions recorded on different days. Looking at the results it is interesting to compare the performance achieved on data sets of different subjects, while applying the same signal processing algorithms. No method achieved good results on all subjects. Especially the session-to-session transfer could have been a source for the occurred problems. Although we provided training data sets from three different days, 2 training data sets were recorded without feedback and just 1 data set with feedback were given, but of course we wanted to see the performance on online data sets with feedback recorded later on different days. The winning algorithms could foster this problem best, but unfortunately their method needed a 2-s delay to the predicted classification output to achieve a better performance. This approach is very useful if offline classification is performed, but for online control applications such a delay causes a lot of problems for the BCI user.

Like in all the BCI competitions before, the data set and the description will continue to be available on the competition web pagehttp://www.bbci.de/competition/iv/. Other researchers interested in EEG single-trial analysis are welcome to test their algorithms on these data sets and to report their results. To imitate competition conditions, all selections of method, features, and model parameters must be confined to the training sets. However, due to the current availability of the labels of the test data and the publication of thorough analyses of these data, future classification results of the competition data cannot fairly be compared to the original submissions.

\section{DATA SET 3}

Data set 3 Directionally modulated MEG activity is provided by S. Waldert, C. Braun, H. Preissl, N. Birbaumer, A. Aertsen, and C. Mehring from Freiburg (Germany), Tübingen (Germany), Trento (Italy), and London (UK). It was recorded in a collaboration of the Institute of Biology I, the Bernstein Center Freiburg (both at the University of Freiburg), the MEG-Center and the Institute of Medical Psychology and Behavioral Neurobiology (both University of Tübingen). It can be freely accessed via http://www.bbci.de/competition/iv/ with the only restriction that the present article as well as (Waldert et al., 2008) is referenced upon any publication of results.

\subsection{BACKGROUND}

Spinal injury patients rank the loss of hand function as one of the most debilitating features of their injury (Anderson, 2004).
An intuitive way to realize a brain-machine interface (BMI) is to access the neural cortical activity that controlled natural hand movements and translate this activity into commands that produce equivalent movements of external effectors (e.g., prosthetic $\mathrm{arm} / \mathrm{hand}$, computer cursor). Such direct motor BMIs require that kinematic parameters of the movement (e.g., movement direction or velocity) can be inferred from the measured neuronal signals.

Online direct motor BMIs have until recently only been realized using spiking activity [single- (SUA) or multi-unit activity (MUA), e.g., Hochberg et al., 2006; Velliste et al., 2008]. Only in the last decade, it has been shown that not only spiking but also neuronal population activity (Figure 21) is tuned to the direction of hand movements. Tuning of neuronal population signals has been demonstrated in several studies using either (a) invasive recordings (local field potentials, LFP; Mehring et al., 2003) and electrocorticogram (ECoG; Leuthardt et al., 2004; Schalk et al., 2007; Pistohl et al., 2008) or (b) non-invasive recordings (electroencephalogram, EEG; Hammon et al., 2008; Waldert et al., 2008; Bradberry et al., 2010; Lv et al., 2010; Wang and Makeig, 2010) and magnetoencephalogram (MEG; Georgopoulos et al., 2005; Waldert et al., 2008; Bradberry et al., 2009; Wang et al., 2010). Very recently, online direct motor BMI control based on decoding movement direction was realized using MEG (Witte et al., 2010) and ECoG (Milekovic et al., 2012).

Among all these studies, intra-cortical recordings (SUA, MUA, LFP) yield the highest amount of information to be extracted about movement direction (Waldert et al., 2009). However, these signals require the implantation of micro-electrodes into the cortex and long-term stable recording of spiking activity remains a difficult problem. Non-invasive EEG and MEG provide less information, but allow for an easy access to human neural activity without any medical risk for the subject. Obviously, current MEG systems cannot be a basis for real-world direct motor BMI. However, MEG is convenient for BMI training and rehabilitation attempts in patients (e.g., in stroke patients; Buch et al., 2008). In this context, optimized algorithms for inferring kinematic parameters from MEG signals could facilitate BMI training and increase the performance of non-invasive direct motor BMIs. To encourage the development of new algorithms, we contributed to the BCI competition IV a data set containing MEG signals recorded while subjects performed hand/wrist movements in four different directions. 


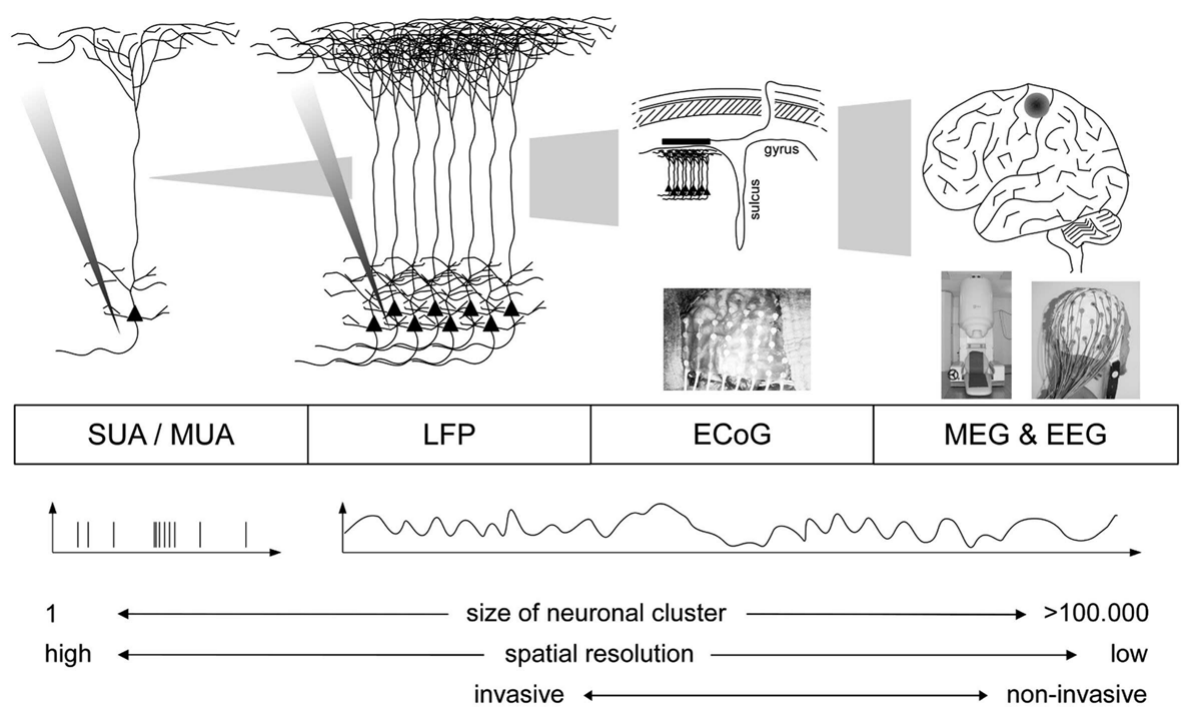

FIGURE 21 | (Data Set 3). Schematic overview of different recording techniques for BMls (from Waldert et al., 2009 with permission).

\subsection{MATERIALS AND SUBJECT}

The data set contained the signals of 10 MEG sensors (VSM MedTech, Vancouver) above central areas measured at $625 \mathrm{~Hz}$ sampling rate during wrist movements of two healthy, right-handed subjects. The subject sat relaxed in an MEG chair, the elbow rested on a pillow to prevent upper arm and shoulder movements, and the head was stabilized by small pillows. The task was to move a joystick from a central resting position toward one of four targets (right, left, forward, backward) using exclusively the right hand and wrist. Movement amplitude was $4.5 \mathrm{~cm}$. In each trial, the target was self-chosen by the subject, i.e., no directional visual cue was provided. Visual trigger signals were presented on a screen in front of the subject to start a trial or to indicate possible time violations. A trial started with the joystick in the center position and the appearance of a gray circle. After a variable delay (1-2 s, Figure 22), the disappearance of the circle indicated the "go" signal (cued movement onset). Then, within $0.75 \mathrm{~s}$ the subject had to start the movement and reach the target. For a trial to be valid, the subject also had to rest at the target for at least $1 \mathrm{~s}$. These time constraints allowed for temporal consistency across trials and the hold period at the target prevented interference of inand outward movements. A red cross was presented continuously for fixation.

\subsection{DATA FORMAT AND PERFORMANCE CRITERIA}

Trials were cut to contain data from $0.4 \mathrm{~s}$ before to $0.6 \mathrm{~s}$ after movement onset. The signals were band-pass filtered $(0.5-100 \mathrm{~Hz})$ and resampled at $400 \mathrm{~Hz}$.

The data were provided as two Matlab "mat"-files, for subject one "S1.mat" and for subject two "S2.mat." Both files contained the variable Info, which provided a detailed description of the data. The second variable, training_data, contained 40 labeled trials per movement direction. These 160 trials were provided to train and evaluate the decoding algorithms. The third variable, test_data, contained 74 (for S-1) or 73 (for S-2) unlabeled trials

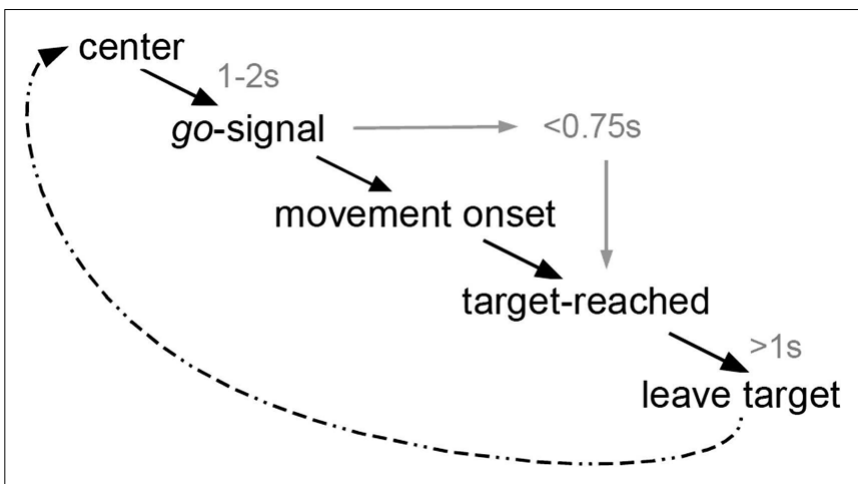

FIGURE 22 | (Data Set 3). Time course of a trial with time constraints (from Waldert et al., 2008 with permission).

in a pseudo-random order. The number of trials per movement direction was unequal but similar. The movement directions of these test trials were not given but had to be predicted from the MEG signals and submitted to the competition. Based on the submitted labels, we calculated the performance of the competitor's algorithms as the percentage of correctly classified trials (decoding accuracy).

\subsection{SUBMISSIONS AND ALGORITHMS}

We received four submissions (ID-1 to ID-4) for this data set. The submission showing best performance was well above chance level for the unlabeled test data. It was submitted by

ID-1: Sepideh Hajipour Sardouie, Mohammad Bagher Shamsollahi. Biomedical Signal and Image Processing Lab (BiSIPL), School of Electrical Engineering, Sharif Univ. of Techn., Tehran, Iran.

The following short summary of the applied algorithms is based on the descriptions provided by the competitors: 
ID-1: A comprehensive set of statistical features, frequencydomain features and wavelet coefficients was extracted from 12 channels (10 real channels plus 2 artificial bipolar channels). The number of features was reduced using a supervised algorithm. Then, a genetic algorithm selected features to optimize the classification accuracy. The classifier consisted of a combination of a linear SVM and LDA. Details of this algorithm are published in (Sardouie and Shamsollahi, 2012).

ID-2: First, a low-pass filter (cutoff $8 \mathrm{~Hz}$ ) was used to filter the time signal. Secondly, the time segment $(0-0.5 \mathrm{~s})$ was selected, that is points 160-360. Third, the first three and five principal components of the abs and angle of the 128 FFT of each channel and each sample were used. Then, Fisher discriminant analysis (FDA) was applied to the frequency features to reduce the dimensionality. Fourthly, the signal were subsampled to $20 \mathrm{~Hz}$. Then, FDA was applied to the time features to reduce dimensionality. Finally, Fisher discriminant functions were used for classification using the combination of time and frequency features.

ID-3: Preprocessing unknown. The feature set consisted of statistical, temporal, parametric and wavelet coefficients and was reduced by PCA and a genetic algorithm. The classifier was a linear SVM.

ID-4: First, a low-pass filter (cutoff $8 \mathrm{~Hz}$ ) was used to filter the time signal. Secondly, the time segment $(0-0.5 \mathrm{~s})$ was selected, that is points 160-360. Third, the first three and five principal components of the abs and angle of the 128 FFT of each channel and each sample were used. Then, FDA was applied to reduce dimensionality. Finally, Fisher discriminant functions were used for classification using the frequency feature.

\subsection{OUTCOME}

All contributors applied either linear SVM, the linear Fisher discriminant analysis (LDA), or a combination of both. Algorithms mainly differed in feature selection. Three competitors (ID-2/3/4) achieved decoding accuracies around chance level of $25 \%$ only for the test data (see Figure 23).

The winner applied a combined linear discriminant analysis (LDA) and linear support vector machine (SVM) on features selected from a large feature set by scattering matrices and a genetic algorithm. The feature set comprised features extracted from the time domain (e.g., AR coefficients, form factor), the frequency domain (e.g., energy in different frequency bands, mean frequency), and the time-frequency domain (wavelet coefficients), but not the low-pass filtered signals that were used in (Waldert et al., 2008). Obtained accuracies on the test data were $59.5 \%$ and $34.3 \%$ for subjects 1 and 2, respectively, and $46.9 \%$ on average.

\subsection{DISCUSSION}

The performance of the competitors algorithms was lower than that of an established decoding algorithm: the application of a regularized linear discriminant analysis (RLDA, also used in Waldert et al., 2008) to the low-pass filtered and resampled signals of the BCI-competition data resulted in a significantly higher average accuracy of $62 \%$ (average across both subjects; $p<0.01$ compared to the competition winner ID-1, Fisher's exact test). Also a linear SVM using the same low-pass filtered signals yielded a

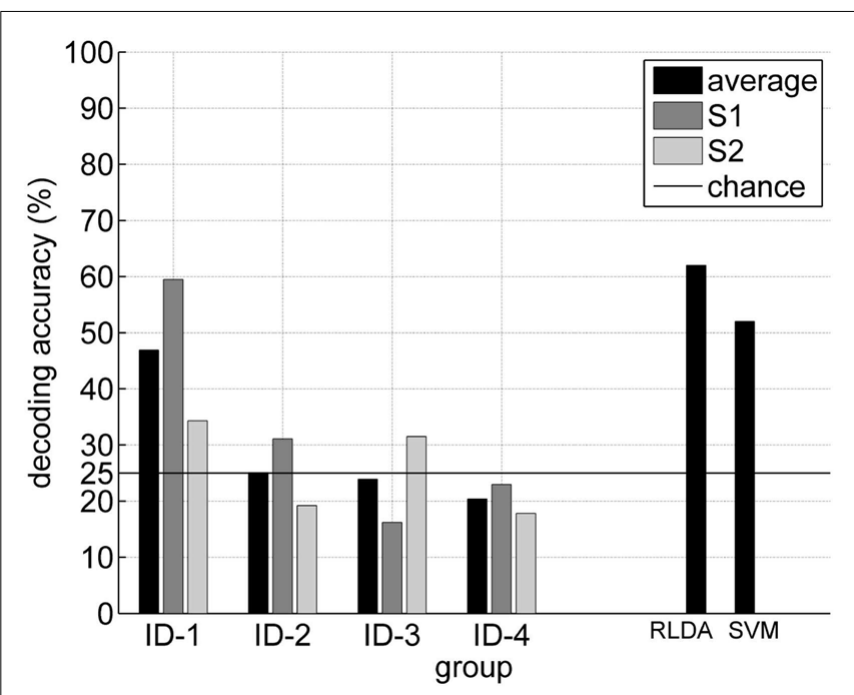

FIGURE 23 | (Data Set 3). Results of the BCl competition IV and, for comparison, the average result of applying a RLDA and linear SVM to the low-pass filtered and resampled activity of the data.

higher average accuracy of 53\% (significantly higher than ID$2 / 3 / 4(p<0.01)$, not significantly higher than ID-1, Fisher's exact test).

ID-2 and ID-4 obtained much higher accuracies on the training data $(98 \%$ and $73 \%)$ than for the test data, which was classified at chance level. This result indicates that the low accuracies for the test data are due to a poor generalization. Possibly the same reason explains the low accuracy for ID-3. However, the performance on the training data was not available for this group.

Compared to the results of the winning group (ID-1), the higher (RLDA) and equal (SVM) accuracies for the two standard linear classifiers without sophisticated feature selection might be explained by the fact that the low-pass filtered activity - which was used in (Waldert et al., 2008) and which was, due to the applied band-pass filter (0.5-100 Hz, see Data Format), also available in the data set contributed to the BCI competition - was not included in the predefined feature set used by the winning group. It is not clear which decoding accuracies could have been achieved with the algorithm of the competition winner if the low-pass filtered activity were included. Especially this signal component contains substantial information about movement kinematics and provides high performance for decoding of neural population signals: LFP (Mehring et al., 2003; Rickert et al., 2005), ECoG (Schalk et al., 2007; Pistohl et al., 2008; Ball et al., 2009), EEG (Waldert et al., 2008; Bradberry et al., 2010; Lv et al., 2010; Wang and Makeig, 2010), and MEG (Jerbi et al., 2007; Waldert et al., 2008; Bradberry et al., 2009; Wang et al., 2010).

\section{DATA SET 4}

Data set 4 Finger Movements in ECoG is provided by K. J. Miller and G. Schalk from Seattle and Albany (USA). The data set can be freely assessed via http://www.bbci.de/competition/iv/ with the only restriction that the present article is referenced upon any publication of results. 


\subsection{MOTIVATION}

The goal for data set 4 of the BCI competition IV was to infer the flexion of individual fingers from signals recorded from the surface of the brain (electrocorticography, ECoG). Compared to EEG, where a higher spatial blurring prevents the detailed localization in single trial on the finger level, the ECoG signals provide a much higher spatial resolution. This data set contained ECoG signals from three subjects, as well as the time courses of the flexion of each of five fingers. The task in the competition was to use the ECoG signals and flexion information in a training set to predict finger flexion for a provided test set. The performance of that prediction was evaluated by calculating the average correlation coefficient $r$ between actual and predicted finger flexion. We received five submissions for this data set. The results of these submissions and recently published studies demonstrate that the timing and degree of finger flexion can be accurately inferred from ECoG in single trials.

Finger flexion is a simple parameter to correlate with an extracted brain state, and thus can serve as a good test bed for algorithm development. There are many potential implications of successful algorithmic decoding of brain states: neural prosthetics, communication devices, handicapped vehicle control (wheelchairs, etc.), and potentially rehabilitation of the brain. The use of motor areas related to hand movements is particularly compelling in this context, because, as an area that is evolutionarily specialized for tool use, it may provide an intuitive basis for controlling prosthetic hands or other manipulandums.

Electrocorticography (ECoG) is the measurement of mesoscale electric potentials $(1-5 \mathrm{~mm})$ from the subdural brain surface. In the data set provided for the BCI competition, all three subjects who participated were epileptic patients receiving ECoG monitoring for the localization of seizure foci (Figure 24). In this setting, ECoG has proven to be a powerful tool for brain-computer interfacing (Leuthardt et al., 2004; Schalk et al., 2008), and capable of augmenting activity in the brain (Miller et al., 2010).

Several features can be extracted from the ECoG data that may correlate with behavior. Motor-related event-related potentials can be extracted from the raw time series (Figure 25D). A running average of the raw signal, termed the local motor potential (LMP; Schalk et al., 2007) has been shown to be informative about task-related brain activity in motor cortex (Schalk et al., 2007; Kubanek et al., 2009; Figure 26). In addition, frequencydomain features have been shown to robustly capture shifts in behavioral state (Crone et al., 1998a,b; Miller et al., 2007). Shifts in different frequency ranges often have different spatial patterns. There is a characteristic decrease in power at low frequencies and increase in power at high frequencies that accompanies movement (Figure 27). The decreases in low frequency power have spatially

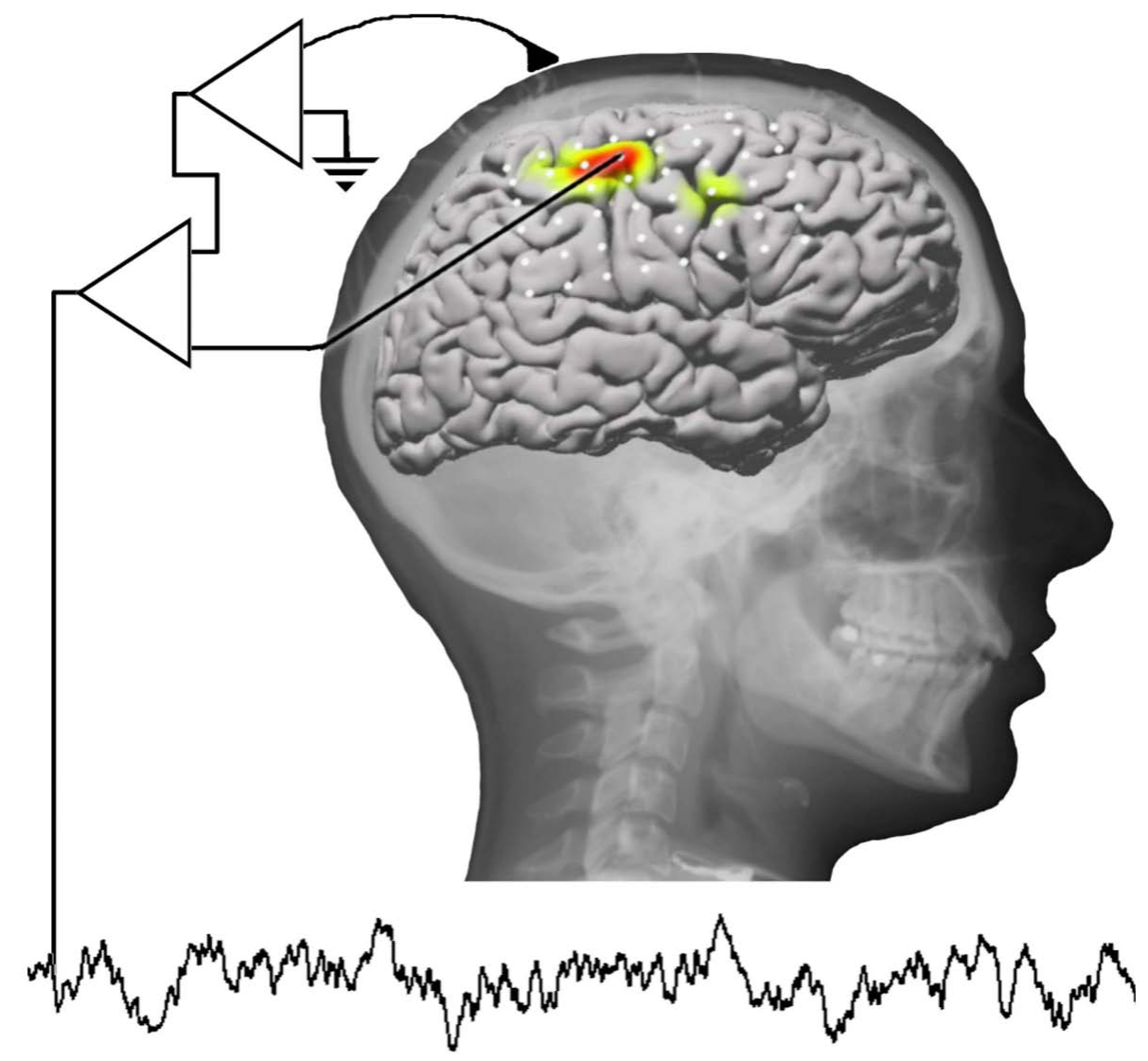

FIGURE 24 | (Data Set 4). The ECoG signals in train_data (time, channel) and test_data (time, channel) were acquired from each electrode with respect to a scalp reference and ground before re-referencing with respect to the common average. 


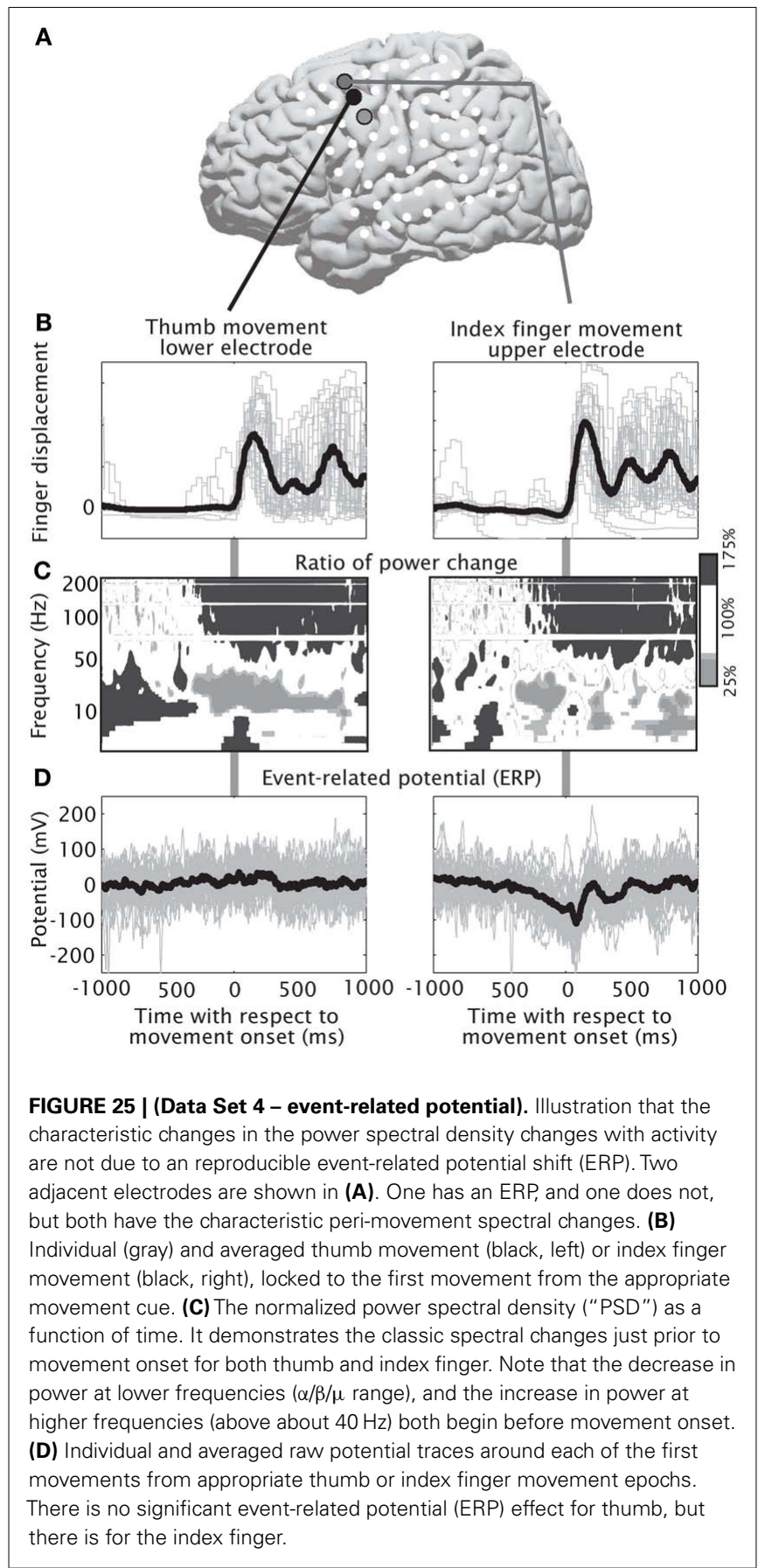

broad distributions, and power increases at high frequencies have spatially more confined distributions (Figure 28). Different fingers have spatially different representations on the brain surface, and this can be used to help distinguish which finger might be moving at any particular time (Figures 28-30).

Time-frequency estimates of power change can serve as robust correlates of behavior (Figures 25 and 27). Recent studies have demonstrated that what had been perceived as a spatially focal high frequency phenomenon was really a reflection of a broadband feature, likely corresponding to average firing potential rate of the

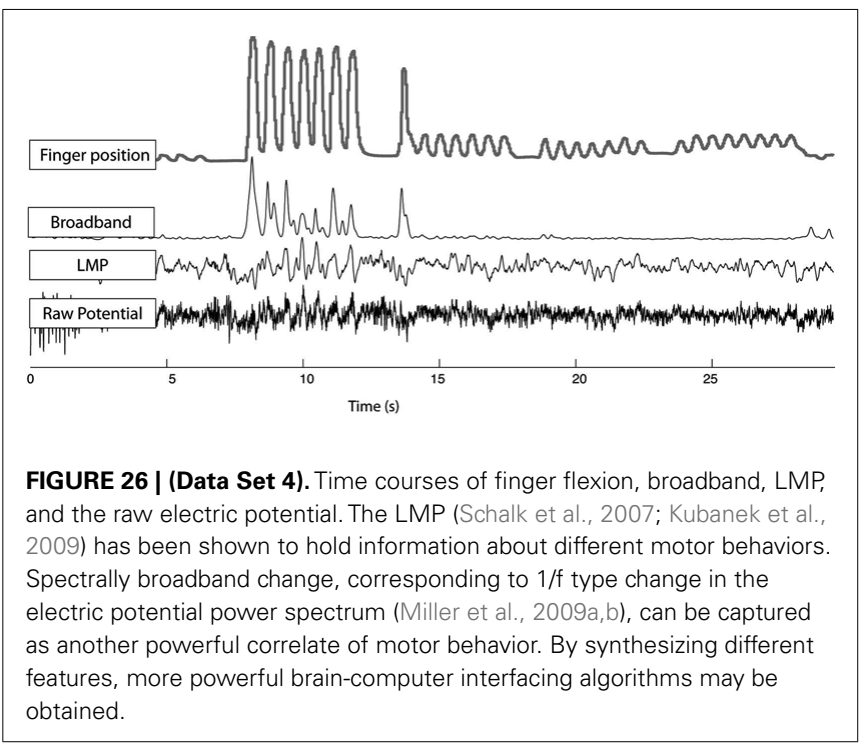

neuronal population beneath the electrode (Miller et al., 2009a,b). When captured, this broadband feature has been demonstrated to be a robust correlate of finger movement at individual sites in motor cortex (Miller et al., 2009b; Figures 26 and 30).

In this competition, participants used different techniques that capitalized on different aspects of these signals to predict the flexion of individual fingers from the ECoG signals.

\subsection{MATERIALS AND SUBJECTS}

The three subjects in the data set were epileptic patients at Harborview Hospital in Seattle, Washington. Each patient had electrode grids placed subdurally on the surface of the brain for the purpose of extended clinical monitoring and localization of seizure foci. Each subject gave informed consent to participate in this study, which was approved by the internal review board (IRB) of Harborview Hospital. All patient data have been anonymized according to IRB protocol in accordance with HIPAA regulations.

\subsubsection{Experimental paradigm}

Signals from the electrode grid were amplified and digitized using Synamps 2 amplifiers (Neuroscan, El Paso, TX, USA). The general-purpose BCI system BCI2000 (Schalk et al., 2004) provided visual stimuli to the patient, acquired brain signals from the Synamps2 system, and also recorded the flexion of individual fingers (on the hand contralateral to the implanted grid) using a data glove (Fifth Dimension Technologies, Irvine, CA, USA). BCI2000 stored the brain signals, the timing of stimulus presentation, and the flexion of each of the fingers in a data file. Data files were converted to MATLAB format for this competition. Each patient had subdural electrode arrays (AdTech, Racine, WI, USA) implanted. Each array contained 4864 platinum electrodes that were configured in $8 \times 6$ or $8 \times 8$ arrangements. The electrodes had a diameter of $4 \mathrm{~mm}(2.3 \mathrm{~mm}$ exposed), $1 \mathrm{~cm}$ inter-electrode distance, and were embedded in silastic. Electrocorticographic (ECoG) signals (i.e., 62, 48, and 64 channels from subjects 1,2 , and 3 , respectively), were 

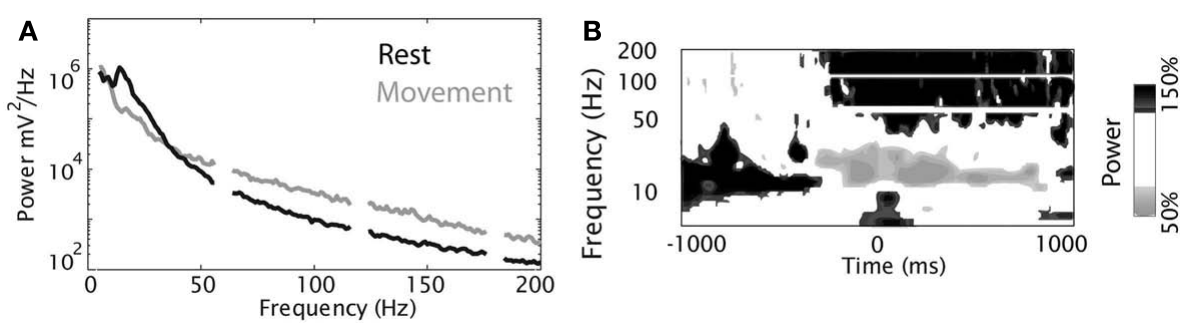

FIGURE 27 | (Data Set 4). Examples of the normalized power spectral density (PSD) of the potential time series around finger flexion. The PSD was calculated from $1 \mathrm{~s}$ windows centered at times of maximum flexion and also during rest. (A) Mean PSD of index finger movement

samples (light trace) and rest samples (black trace). (B) Average time-varying PSD (scaled as percentage of mean power at each frequency) with respect to first index finger movement from each movement cue.

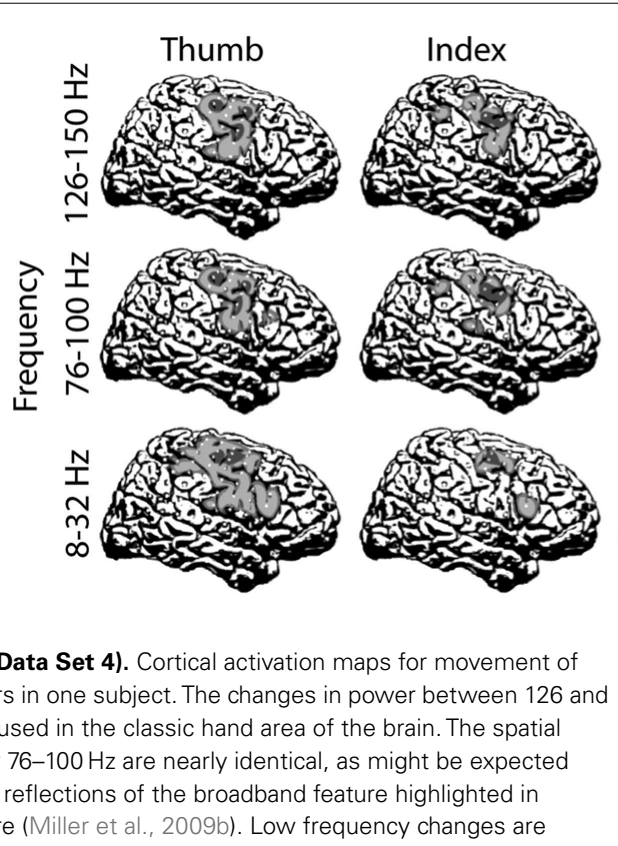

FIGURE 28 | (Data Set 4). Cortical activation maps for movement of different fingers in one subject. The changes in power between 126 and $150 \mathrm{~Hz}$ are focused in the classic hand area of the brain. The spatial distribution for $76-100 \mathrm{~Hz}$ are nearly identical, as might be expected since both are reflections of the broadband feature highlighted in recent literature (Miller et al., 2009b). Low frequency changes are

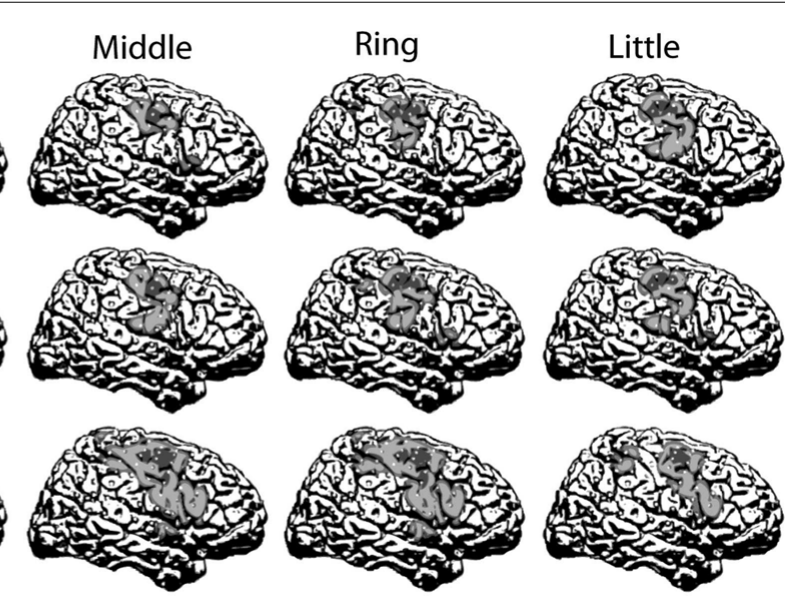

spatially much more broad, corresponding to fluctuations in the classic motor rhythms. Figure $\mathbf{2 9}$ shows that the spatial representations for high frequencies are very different for different finger movement types, within a general hand region. Electrode positions are shown with white dots, and power change with light and dark gray patches on the brain surface. acquired with respect to a scalp reference and ground (Figure 24), band-pass filtered between 0.15 and $200 \mathrm{~Hz}$, and sampled at $1000 \mathrm{~Hz}$.

\subsubsection{Protocol}

The subjects were cued to move a particular finger by displaying the corresponding word (e.g., "thumb") on a computer monitor placed at the bed-side (Figure 30). Each cue lasted $2 \mathrm{~s}$ and was followed by a 2-s rest period during which the screen was blank. During each cue, the subjects typically moved the requested finger 3-5 times. This number varied across subjects and fingers. There were 30 movement stimulus cues for each finger (i.e., a total of 150 cue presentations and about 90-150 flexions of each finger); stimulus cues were interleaved randomly. This experiment lasted $10 \mathrm{~min}$ for each subject.

Subsequent offline analyses showed that ring (4th) finger movements were correlated with either middle (3rd) or little (5th) finger movements. Thus, while this ring finger position was included with the training data, it was not used for evaluation.

\subsection{DATA FORMAT}

The data for each subject was contained in a separate MATLAB file that was named "subX_comp.mat" where " $X$ " denotes the subject number. Each file contained three variables:

- "train_data" - this variable, in time $\times$ channels, gave the first 2/3 (6 min, $40 \mathrm{~s}$ ) of recorded ECoG signals (400,000 samples at $1 \mathrm{kHz}$ sampling rate per channel) from the specified experiment, for every channel.

- "train_dg" - this variable, in time $\times$ finger was the first $2 / 3$ (6 min, $40 \mathrm{~s}$ ) of recorded finger position [thumb - index middle - ring - little; 400,000 samples (super-sampled to $1 \mathrm{kHz}$ ) per finger] for the associated experiment.

- "test_data" - this variable, in time $\times$ channels, gave the last 1/3 (3 min, 20 s) of recorded ECoG signals (200,000 samples at $1 \mathrm{kHz}$ sampling rate per channel) from the specified experiment, for every channel. These data were used to predict the final $1 / 3$ ( $3 \mathrm{~min}, 20 \mathrm{~s}$ ) of recorded finger position (thumb - index - middle - ring - little) for the associated experiment. 
The channel order was scrambled so that the prediction task in the competition was restricted to algorithmic optimizations only.

\subsection{ChALLENGE}

Each participating group submitted three files titled "sub1_eval," "sub2_eval," and "sub3_eval," corresponding to subjects 1-3, respectively. Each of these contained a single variable, "eval_dg," with dimensions $200,000 \times 5$ :
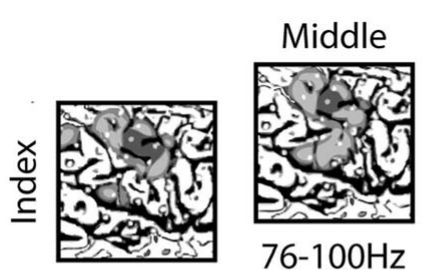

$76-100 \mathrm{~Hz}$
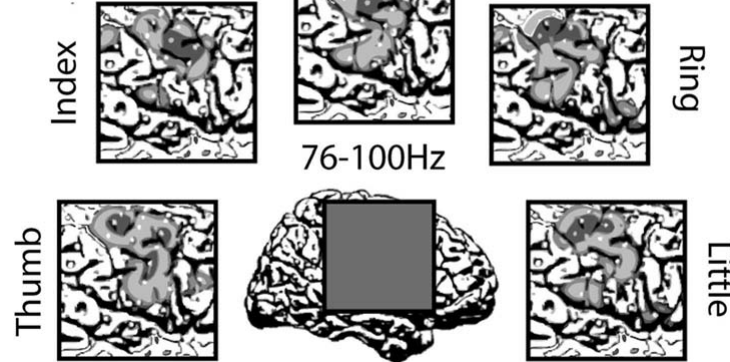

FIGURE 29 | (Data Set 4). A blow-up of the sensorimotor region for high frequencies from Figure 28. Note that this variability across electrodes allows for robust segregation of different finger movements during classification.
- "eval_dg" - this variable, in time $\times$ channels, gave the last $1 / 3$ (3 min, 20s) of predicted finger flexion for each of the five fingers (thumb - index - middle - ring - little) for the associated experiment (200,000 samples per finger).

The evaluation criteria was as follows: for each subject, the received variable "eval_dg" was compared with the actual finger positions in "test_dg," which was withheld. We calculated the correlation coefficient $r$ between the actual and the predicted finger flexions for each subject and finger. We did not calculate the correlation coefficient for the 4th (ring) finger, because the flexion of this finger was typically correlated with the flexion of the 3rd (middle) or 5th (little) finger. The final score was calculated as the arithmetic mean of the 12 correlation coefficients ( 4 per subject, 3 subjects). The submission with the highest score won the competition.

\subsection{SUBMISSIONS AND ALGORITHMS}

Five groups submitted a contribution (S-1 to $S-5)$, with three of them (S-1, S-2, S-4) showing a performance well above chance level on the unseen test set.

S-1: Remi Flamary, Alain Rakotomamonjy, LITIS INSA de Rouen, France

S-2: Nanying Liang and Laurent Bougrain, Cortex Team, Research Centre INRIA, Nancy-Grand Est, France

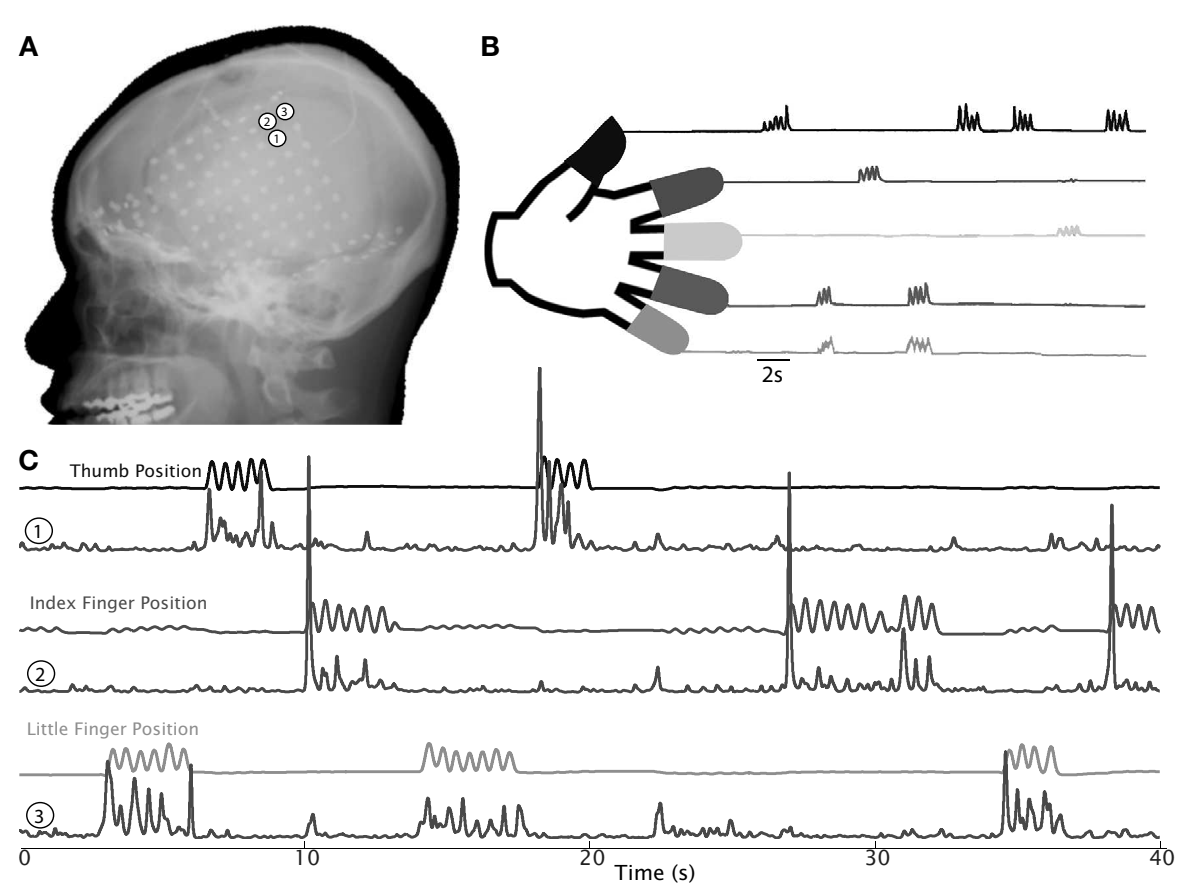

FIGURE 30 | (Data Set 4). Time course of ECoG in adjacent electrodes reveals individual digit representation. (A) X-ray of the ECoG array in situ, with three electrodes labeled, corresponding to the numbers in (C). (B) Flexion time course of each finger. (C) Projections of the time-frequency representation to broadband spectral change (Miller et al., 2009b). Each electrode is specifically and strongly correlated with one movement type $(r=0.46$ for broadband from electrode 1 with thumb position; $r=0.47$ for electrode 2 with index finger; $r=0.29$ for electrode 3 with little finger; cross-combinations had a mean correlation of -0.09 , indicating light hyperextension of other fingers while flexing the appropriate finger in this subject), over 10 min of continuous data $\left(3.6 \times 10^{6}\right.$ samples). 


\section{S-4: Mathew Salvaris, University of Essex, Colchester, UK}

The following short summary of the applied algorithms is based on the descriptions provided by the competitors:

\section{S-1}

Flamary and Rakotomamojy employed a switching model to predict finger flexion. This method assumed that the output flexion of the fingers is linear and that the transfer function between ECoG signals and finger position depended on an internal state $k$ that represents the finger moving (1-5) or no finger moving at all (6). They used ridge regression to compute the transfer function and sparse linear regression to derive the state estimation. In brief, signals were first down-sampled by a factor of 4 . The features for the linear transfer functions were obtained with a Savitsky-Golay filter $(0.4 \mathrm{~s}, 3 \mathrm{rd}$ order $)$. The features used for the state estimator were AR coefficients computed on a moving window of 300 points. Once the internal state was estimated, finger flexion was computed by multiplying the features at a time $t$ by the linear transfer function corresponding to the state $k$ at time $t$.

\section{S-2}

Liang and Bougrain first extracted, from each location, the timevarying activity in three frequency bands: 1-60, 60-100, and 100$200 \mathrm{~Hz}$. Then, the power in each bin was accumulated in $40 \mathrm{~ms}$ time bins. The size of the time bin was chosen so that the resulting amplitude modulation feature inputs had the same sampling rate (i.e., $25 \mathrm{~Hz}$ ) as that of the finger flexion values. Initial evaluations found that each finger flexion was correlated to features from only two or three particular locations. Therefore, features were automatically selected (separately for each finger and subject) using a stepwise feature selection procedure based on the train and validation method (i.e., 2/3 of train data were used for training and $1 / 3$ for validation). The resulting features were then submitted to a Wiener filter with 25 tap-delays (i.e., using the present input and the previous 1-s inputs for predicting the present finger flexion).

\section{S-4}

Salvaris first re-referenced signals to the common average, and then down-sampled signals to $500 \mathrm{~Hz}$. Bandpower features were extracted by wavelet packets with sym 9 wavelet and the average of the time series. Features were then selected using WEKA's CFS algorithm. The selected features were used to train the SVR algorithm implemented in LibSVM. The parameters for SVR were tuned through 5-fold cross validation. The resulting SVR model was then used to classify the test data.

\subsection{RESULTS}

The goal of this portion of the competition was to predict finger flexion for four of the five fingers on a test set ( $3 \mathrm{~min} 20 \mathrm{~s}$ ) using a classifier that was trained on a training set $(6 \mathrm{~min} 40 \mathrm{~s})$. The fidelity of the prediction was assessed by computing the correlation coefficient between the actual finger flexion values and the submitted finger flexion values. The result of a particular submission was the arithmetic mean of 12 correlation coefficients (i.e., 3 subjects and 4 fingers).

Two of the five submissions achieved particularly strong predictions (see Table 5). Nanying Liang and Laurent Bougrain achieved
Table 5 | (Data Set 4). Performance of the five submissions.

\begin{tabular}{lc}
\hline Submission & $\mathbf{r}$ \\
\hline S-2 & 0.46 \\
S-1 & 0.42 \\
S-4 & 0.27 \\
S-3 & 0.10 \\
S-5 & 0.05 \\
\hline
\end{tabular}

an average correlation coefficient of 0.46 , and thereby won this competition. The runner-up contribution of Flamary and Rakotomamojy performed similarly well with a correlation coefficient of 0.42. Details of the two approaches are described in Flamary and Rakotomamonjy (2012) and Liang and Bougrain (2012).

While it is difficult to assess the difference in performance between the different methods, it is interesting that methods that are similar in simplicity to those used in Schalk et al. (2007) and Kubanek et al. (2009) can reliably and robustly estimate finger flexion from ECoG signals. That being the case, it may also be possible that more sophisticated methods that explicitly incorporate physiological or physical constraints in the computational model might further improve performance.

\section{DISCUSSION}

The BCI competition was created in order to support the development of algorithmic solutions for typical BCI problems. Does it live up to its promise? The following sections attempt to give an answer to the various aspects of this question.

\subsection{REST CLASS PROBLEM REMAINS A CHALLENGE}

Moving from an artificial lab situation to the every-day use of a $\mathrm{BCI}$ introduces a new challenge: periods of non-control, where a BCI user is voluntarily switching to another (non-BCI) action or is involuntarily distracted from the control interface. The distractor can be another active task (e.g., communication via a different channel, the perception and processing of content, reasoning about a decision to take) or simply taking a rest. The basic problem about rest class detection in general is, that the resting state is not welldefined at all, and thus there is no reliable training data available that can be used to calibrate the BCI system.

In this competition, the detection of such a rest class was challenged with data set 1 . The results for this motor imagery data set revealed, that most competitors had problems in correctly identifying time periods of the rest class. Even considering the performance of the competition winner (Figure 6) there remains the wish for further improvement.

\subsection{TRANSFERABILITY TO ONLINE BCIS}

The winning methods of this or earlier competitions are not necessarily transferable to be used in an online closed-loop BCI system. While the runtimes of algorithms are not a real limitation, noncausal filters and time delays are problematic. As an example, the winning algorithm of data set $2 \mathrm{~b}$ predicted the class labels quite accurately, but introduced a delay of $2 \mathrm{~s}$ during the preprocessing eye artifacts. This is a trade-off that has to be considered for each specific application. 
High robustness and generalizability of a winning algorithm is another characteristic, that supports the applicability of an algorithm for the feedback case. Comparable to earlier BCI competitions, again variants of CSP ruled the rankings. Of special interest is the outcome, that the Singapore group has scored exceptionally high for several of the data sets. As the submitted algorithms of this group used similar concepts, this is a strong indicator for robustness.

As some of the winning algorithms of earlier competitions have indeed been adopted into the standard canon for BCI online control, we believe, that this will also happen for some algorithms of the present competition.

\subsection{USEFULNESS OF SYNTHETIC DATA}

The most reliable way of testing new algorithmic ideas for BCI is to implement them in an online experiment, if possible with users matching the target group. But even when testing with healthy users, the testing effort is huge and can not be invested for every change of the algorithmic model.

Synthetic EEG data as presented in data set 1-artificial might offer a partial remedy to this problem. It has proven to be realistic in the sense that the submitted algorithms performed very similar on the synthetic and the real EEG data. As it is cheap to generate a large amount of this data, at least initial algorithmic test beds can be based on it. Precautions, of course, have to be taken in order to avoid that the priors used for the EEG generation are not known or explicitly exploited by the algorithms under test and their creators.

As simulated BCI classifier output has already successfully been applied for the fine-tuning of BCI user interfaces (Quek et al., 2011), the next step is on the horizon: to use simulated EEG that certainly only to a limited extend - models the user behavior, in order to test BCI systems online in a closed loop.

\section{FURTHER TOPICS CONCERNING FUTURE COMPETITIONS}

Due to the development of the field of BCI, new data analytic problems were identified, that are suitable for addressing them in a BCI competition.

\subsection{WIRELESS AND DRY EEG SIGNALS}

We currently observe the upcoming of easy-to-mount dry electrode caps as either research prototypes (Popescu et al., 2007; Gargiulo et al., 2010; Luo and Sullivan, 2010; Saab et al., 2011; Zander et al., 2011) or purchasable products (e.g., Sahara dry cap by gTec, Mindset by NeuroSky, or Emotiv cap). As some of them provide wireless transmission protocols, they open up the possibility to monitor the acting brain during real-life situations rather than under artificial lab conditions.

The signals of these dry electrodes, however, currently still suffer from a number of artifacts, which are typically much weaker or not present at all in wet electrode recordings. Examples are inductive artifacts by persons moving in the same room, drifts and saturation effects, or friction artifacts upon electrode movements. While an overall higher noise level of dry electrodes might be difficult to overcome, some artifacts might be alleviated by suitable data processing. A future BCI data competition should thus include a number of dry sensor data sets to determine the most effective approaches.

\subsection{NON-STATIONARITY}

Severe for the use of dry EEG sensors, but not restricted to this signal type, is the problem of non-stationarity in brain signals. In the context of BCI, it is mostly observed during the transition from the initial calibration phase to the online use of a BCI (Shenoy et al., 2006; Sugiyama et al., 2007), but also within periods of online use, where no obvious change of the task or paradigm takes place.

The reasons for non-stationarity in brain data can range from external noise, over effects caused by high dimensionality and robust estimation problems (Sannelli et al., 2008; Abrahamsen and Hansen, 2011; task-unrelated) changes in the background brain activity of BCI users (e.g., due to fatigue or artifacts; Winkler et al., 2011, learning effects or adaptive behavior of the users; Ramsey et al., 2009, or even co-adaptation of users and the BCI system Vidaurre et al., 2011).

Non-stationarity can sometimes be observed even by bare eye in the raw data, where it is present in the form of slow drifts, changes in oscillatory sources, or changes in the noise level of electrodes. If processed with an automatic classification or regression method as in BCI, this processing can be harmed also by subtle bias shifts, covariance drifts or changes of the covariance structure, or even more complex changes of the data distributions.

Although a number of methods have been proposed to mitigate this problem either by finding a global stable subspace for the data representation (Krauledat et al., 2007; Blankertz et al., 2008a; von Bünau et al., 2009; Wojcikiewicz et al., 2011), or by adapting the online processing to compensate for ongoing changes (see Vidaurre and Schlögl, 2008; Blankertz and Vidaurre, 2009; Sannelli et al., 2011) for adaptation in motor-related tasks, and (Dähne et al., 2011) for adaptation in ERP paradigms), it still is the source of major problems in the online use of BCI. This qualifies the problem of non-stationarity for becoming a target in future BCI competitions.

\subsection{MULTIMODAL SIGNALS/HYBRID BCls}

Considering the predominant use of non-invasive BCI systems, it is worth to briefly review the development of BCI performance (e.g., in terms of communication rates) over time. On the positive side, new BCI systems based on external stimuli have recently been reported, that employed novel paradigms for auditory (Schreuder et al., 2010, 2011; Höhne et al., 2011) and for visual ERP setups (Liu et al., 2010; Acqualagna and Blankertz, 2011; Schaeff et al., 2011; Tangermann et al., 2011; Treder et al., 2011). They improve over long-used standard stimulation paradigms or can provide solutions for patients that have lost eye gaze control. In contrary, the improvements reported for BCI systems based on motor imagery and ERD/ERS effects have been slower over the last years, despite of a drastic initial performance boost which was made possible by the introduction of machine learning methods (Blankertz et al., 2003, 2011; Schröder et al., 2003).

The next boost of BCI performance can be expected for paradigms, that are able to combine independent information from different sources in order to improve the BCI control quality over the level of a traditional single-source BCI. In an ERP setup, such approaches could combine stimuli of different sensory modalities (Aloise et al., 2007). In motor imagery, the use of ERD/ERS effects together with slower motor-related potentials (Dornhege 
et al., 2004) can increase information rates. Abstracting this concept to the next level, EEG signals could be combined with other brain signal sources like fNIRS (Fazli et al., 2012), with non-neural but physiological signals (e.g., heart rate variability, galvanic skin resistance, pupil dilation, etc.) or in a hybrid setup (Millán et al., 2010; Pfurtscheller et al., 2010; Müller-Putz et al., 2011) e.g., in combination with non-BCI assistive technology.

We currently observe an expansion of BCI technology to other fields. As it gives access to the real-time monitoring of mental states (Müller et al., 2008), it is interesting for neuro-ergonomic interface- and product design (Blankertz et al., 2010; Porbadnigk et al., 2010). Furthermore it starts becoming a tool for the neurosciences, where the use of multiple sources of information is an inviting possibility. All these fields can profit from processing methods, that are capable of linking brain data with behavioral data or with non-neural physiological signal types.

The challenge in processing signals from multiple sources is to represent, combine and converge information in a way, that is independent of different sampling rates (Bießmann et al., 2009; Biessmann et al., 2011), SNR-levels or varying levels of nonstationarity. It is a great challenge with multiple facets. A next BCI competition could contribute to the exploration of at least a few of these aspects.

\section{PERFORMANCE BASELINE FOR PARTICIPATION}

The results of competition IV and the three past competitions have shown, that the number of entries per data set varies to a large extent, probably due to differing levels of effort that have to be invested. Participants tend to submit more entries for standard learning problems, e.g., classification problems where the percentage of correct classifications is the metric of choice. Non-standard learning problems, even though representing important problems in the field of BCI, tend to gain less attention.

As the success of a participant is finally expressed as a rank among all submitted entries, the small sample ranking can

\section{REFERENCES}

Abrahamsen, T. J., and Hansen, L. K. (2011). A cure for variance inflation in high dimensional kernel principal component analysis. J. Mach. Learn. Res. 12, 2027-2044.

Acqualagna, L., and Blankertz, B. (2011). A gaze independent speller based on rapid serial visual presentation. Conf. Proc. IEEE Eng. Med. Biol. Soc. 2011, 4560-4563.

Aloise, F., Lasorsa, I., Schettini, F., Brouwer, A., Mattila, D., Babiloni, F. Salinari, S., Marciani, M., and Cincotti, F. (2007). Multimodal stimulation for a P300-based BCI. Int. J. Bioelectromagn. 9, 128-130.

Anderson, K. (2004). Targeting recovery: priorities of the spinal cordinjured population. J. Neurotrauma 21, 11371-11383.

Ang, K., Chin, Z. Y., Zhang, H., and Guan, C. (2008). "Filter bank common spatial pattern (FBCSP) in brain-computer interface," in
Proceedings of the IEEE International Joint Conference on Neural Networks (IJCNN'08), Hong Kong, 2391-2398.

Ang, K., and Quek, C. (2006). "Rough set-based neuro-fuzzy system," in Proceedings of the IEEE International Joint Conference on Neural Networks (IJCNN'06), Vancouver, 742-749.

Ang, K. K., Chin, Z. Y., Wang, C., Guan, C., and Zhang, H. (2012). Filter bank common spatial pattern algorithm on BCI competition iv datasets $2 \mathrm{a}$ and $2 \mathrm{~b}$. Front. Neurosci. 6:39. doi:10.3389/fnins.2012.00039

Ball, T., Schulze-Bonhage, A., Aertsen, A., and Mehring, C. (2009). Differential representation of arm movement direction in relation to cortical anatomy and function. J. Neural Eng. 6, 016006

Bießmann, F., Meinecke, F. C., Gretton, A., Rauch, A., Rainer, G., Logothetis, N., and Müller, K.-R. (2009). Temporal kernel canonical correlation

potentially be misleading with respect to the overall quality of even the best-ranked entry. For this reason, it is planned to introduce a performance threshold in future BCI competitions. It will be determined based on the test data. All entries have to pass this threshold before they can enter the official ranking. The threshold is to be defined by the data issuing group and should represent the state-of-the-art performance that can be gained with established analysis methods. The threshold is published together with the performance metric and with a short description of the standard method that leads to this performance.

We think that this action will contribute toward assessing the absolute quality of a competition entry rather than the relative quality only. On the long run the introduction of a threshold can increase the perceived reliability of novel methods brought to the BCI community via a BCI competition, and speed up their adoption by BCI practitioners.

\section{ACKNOWLEDGMENTS}

The studies were in part or completely supported by the Bundesministerium für Bildung und Forschung (BMBF), Fkz 01IB001A, 01GQ0850, by the German Science Foundation (DFG, contract MU 987/3-2), by the European ICT Programme Projects FP7-224631 and 216886, the World Class University Program through the National Research Foundation of Korea funded by the Ministry of Education, Science, and Technology (Grant R3110008), the US Army Research Office [W911NF-08-1-0216 (Gerwin Schalk) and W911NF-07-1-0415 (Gerwin Schalk)] and the NIH [EB006356 (Gerwin Schalk) and EB000856 (Gerwin Schalk), the WIN-Kolleg of the Heidelberg Academy of Sciences and Humanities, German Federal Ministry of Education and Research grants 01GQ0420, 01GQ0761, 01GQ0762, and 01GQ0830, German Research Foundation grants 550/B5 and C6, and by a scholarship from the German National Academic Foundation. This paper only reflects the authors' views and funding agencies are not liable for any use that may be made of the information contained herein.

analysis and its application in multimodal neuronal data analysis. Mach. Learn. 79, 5-27.

Biessmann, F., Plis, S., Meinecke, F. C. Eichele, T., and Müller, K.-R. (2011). Analysis of multimodal neuroimaging data. IEEE Rev. Biomed. Eng. 4, 26-58.

Bijma, F., de Munck, J., Huizenga, H., and Heethaar, R. (2003). A mathematical approach to the temporal stationarity of background noise in MEG/EEG measurements. $\mathrm{Neu}$ roimage 20, 233-243.

Blanchard, G., and Blankertz, B. (2004). BCI competition 2003 - data set IIa: spatial patterns of self-controlled brain rhythm modulations. IEEE Trans. Biomed. Eng. 51, 1062-1066.

Blankertz, B., Dornhege, G., Schäfer, C. Krepki, R., Kohlmorgen, J., Müller, K. -R., Kunzmann, V., Losch, F., and Curio, G. (2003). Boosting bit rates and error detection for the classification of fast-paced motor commands based on single-trial EEG analysis. IEEE Trans. Neural Syst. Rehabil. Eng. 11, 127-131.

Blankertz, B., Kawanabe, M., Tomioka, R., Hohlefeld, F., Nikulin, V., and Müller, K.-R. (2008a). "Invariant common spatial patterns: alleviating nonstationarities in brain-computer interfacing," in Advances in Neural Information Processing Systems 20, eds J. Platt, D. Koller, Y. Singer, and S. Roweis (Cambridge, MA: MIT Press), 113-120.

Blankertz, B., Tomioka, R., Lemm, S., Kawanabe, M., and Müller, K.-R. (2008b). Optimizing spatial filters for robust EEG single-trial analysis. IEEE Signal Process. Mag. 25, 41-56.

Blankertz, B., Lemm, S., Treder, M. S., Haufe, S., and Müller, K.-R. (2011). Single-trial analysis and classification of ERP components - a tutorial. Neuroimage 56, 814-825. 
Blankertz, B., Müller, K.-R., Curio, G., Vaughan, T. M., Schalk, G., Wolpaw, J. R., Schlögl, A., Neuper, C., Pfurtscheller, G., Hinterberger, T., Schröder, M., and Birbaumer, N. (2004). The BCI competition 2003: progress and perspectives in detection and discrimination of EEG single trials. IEEE Trans. Biomed. Eng. 51, 1044-1051.

Blankertz, B., Müller, K.-R., Krusienski, D., Schalk, G., Wolpaw, J. R., Schlögl, A., Pfurtscheller, G., Millán Jdel, R., Schröder, M., and Birbaumer, N. (2006). The BCI competition III: validating alternative approachs to actual $\mathrm{BCI}$ problems. IEEE Trans. Neural Syst. Rehabil. Eng. 14, 153-159.

Blankertz, B., Tangermann, M., Vidaurre, C., Fazli, S., Sannelli, C., Haufe, S., Maeder, C., Ramsey, L. E., Sturm, I., Curio, G., and Müller, K.-R. (2010). The Berlin brain-computer interface: non-medical uses of BCI technology. Front. Neurosci. 4:198. doi:10.3389/fnins.2010.00198

Blankertz, B., and Vidaurre, C. (2009). Towards a cure for BCI illiteracy: machine-learning based co-adaptive learning. BMC Neurosci. 10(Suppl. 1), P85. doi:10.1186/1471-2202-10S1-P85

Bostanov, V. (2004). BCI competition 2003 - data sets Ib and IIb: feature extraction from event-related brain potentials with the continuous wavelet transform and the $\mathrm{t}$-value scalogram. IEEE Trans. Biomed. Eng. 51, 1057-1061.

Bradberry, T., Gentili, R., and Contreras-Vidal, J. (2010). Reconstructing three-dimensional hand movements from noninvasive electroencephalographic signals. $J$. Neurosci. 30, 3432-3437.

Bradberry, T., Rong, F., and ContrerasVidal, J. (2009). Decoding centerout hand velocity from MEG signals during visuomotor adaptation. Neuroimage 47, 1691-1700.

Brunner, C., Billinger, M., Vidaurre, C., and Neuper, C. (2011). A comparison of univariate, vector, bilinear autoregressive, and band power features for brain-computer interfaces. Med. Biol. Eng. Comput. 49, 1337-1346.

Brunner, C., Naeem, M., Leeb, R., Graimann, B., and Pfurtscheller, G. (2007). Spatial filtering and selection of optimized components in four class motor imagery EEG data using independent components analysis. Pattern Recognit. Lett. 28, 957-964.

Buch, E., Weber, C., Cohen, L., Braun, C., Dimyan, M., Ard, T., Mellinger, J., Caria, A., Soekadar, S., Fourkas,
A., and Birbaumer, N. (2008). Think to move: a neuromagnetic braincomputer interface (BCI) system for chronik stroke. Stroke 39, 910-917.

Carlqvist, H., Nikulin, V., Strömberg, J., and Brismar, T. (2004). Amplitude and phase relationship between alpha and beta oscillations in the human electroencephalogram. J. Med. Biol. Eng. Comput. 43, 599-607.

Crone, N. E., Miglioretti, D. L., Gordon, B., and Lesser, R. P. (1998a). Functional mapping of human sensorimotor cortex with electrocorticographic spectral analysis. II. Event-related synchronization in the gamma band. Brain 121(Pt 12), 2301-2315.

Crone, N., Miglioretti, D., Gordon, B., Sieracki, J., Wilson, M., Uematsu, S., and Lesser, R. (1998b). Functional mapping of human sensorimotor cortex with electrocorticographic spectral analysis. I. Alpha and beta event-related desynchronization. Brain 121, 2271.

Dähne, S., Höhne, J., and Tangermann, M. (2011). "Adaptive classification improves control performance in ERP-based BCIs," in Proceedings of the 5th International BCI Conference, Graz, 92-95.

Millán, J. D., Rupp, R., Müller-Putz, G., Murray-Smith, R., Giugliemma, C., Tangermann, M., Vidaurre, C., Cincotti, F., Kübler, A., Leeb, R., Neuper, C., Müller, K.-R., and Mattia, D. (2010). Combining braincomputer interfaces and assistive technologies: state-of-the-art and challenges. Front. Neurosci. 4:161. doi:10.3389/fnins.2010.00161

Dornhege, G., Blankertz, B., Curio, G., and Müller, K.-R. (2004). Boosting bit rates in non-invasive EEG single-trial classifications by feature combination and multi-class paradigms. IEEE Trans. Biomed. Eng. 51, 993-1002.

Fatourechi, M., Bashashati, A., Ward, R. K., and Birch, G. E. (2007). EMG and EOG artifacts in brain computer interface systems: a survey. Clin. Neurophysiol. 118, 480-494.

Fazli, S., Mehnert, J., Steinbrink, J., Curio, G., Villringer, A., Müller, K. R., and Blankertz, B. (2012). Enhanced performance by a hybrid NIRS-EEG brain computer interface. Neuroimage 59, 519-529.

Flamary, R., and Rakotomamonjy, A. (2012). Decoding finger movements from ECoG signals using switching linear models. Front. Neurosci. 6:29. doi:10.3389/fnins.2012.00029

Freeman, W. (2004a). Origin, structure, and role of background EEG activity. Part 1. Analytic amplitude. Clin. Neurophysiol. 115, 2077-2089.

Freeman, W. (2004b). Origin, structure, and role of background EEG activity. Part 2. Analytic phase. Clin Neurophysiol. 115, 2089-2107.

Freeman, W. (2005). Origin, structure, and role of background EEG activity. Part 3. Neural frame classification. Clin. Neurophysiol. 116, 1118-1129.

Freeman, W. (2006). Origin, structure, and role of background EEG activity. Part 4. Neural frame simulation. Clin. Neurophysiol. 117, 572-589.

Galan, F., Oliva, F., and Guardia, J. (2007). Using mental tasks transitions detection to improve spontaneous mental activity classification. Med. Biol. Eng. Comput. 45, 603-609.

Gargiulo, G., Calvo, R. A., Bifulco, P., Cesarelli, M., Jin, C., Mohamed, A., and van Schaik, A. (2010). A new EEG recording system for passive dry electrodes. Clin. Neurophysiol. 121, 686-693.

Georgopoulos, A., Langheim, F., Leuthold, A., and Merkle, A. (2005). Magnetoencephalographic signals predict movement trajectory in space. Exp. Brain Res. 167, 132-135.

Georgopoulos, A. P., Kalaska, J. F., Caminiti, R., and Massey, J. T. (1982). On the relations between the direction of two-dimensional arm movements and cell discharge in primate motor cortex. J. Neurosci. 2 , 1527-1537.

Geselowitz, D. (1967). On bioelectric potentials in an inhomogeneous volume conductor. Biophys. J. 7, 1-11.

Hammon, P., Makeig, S., Poizner, H., Todorov, E., and de Sa, V. (2008). Predicting reaching targets from human EEG. IEEE Signal Process. Mag. 25, 69-77.

Haufe, S., Treder, M. S., Gugler, M. F., Sagebaum, M., Curio, G., and Blankertz, B. (2011). EEG potentials predict upcoming emergency brakings during simulated driving. J. Neural Eng. 8, 056001.

Henninghausen, E., Heil, M., and Rösler, F. (1993). A correction method for dc drift artifacts. Electroencephalogr. Clin. Neurophysiol. 86, 199-204.

Hochberg, L., Serruya, M., Friehs, G., Mukand, J., Saleh, M., Caplan, A., Branner, A., Chen, D., Penn, R., and Donoghue, J. (2006). Neuronal ensemble control of prosthetic devices by a human with tetraplegia. Nature 442, 164-171.

Höhne, J., Schreuder, M., Blankertz, B., and Tangermann, M. (2011). A novel 9-class auditory ERP paradigm driving a predictive text entry system. Front. Neurosci. 5:99. doi:10.3389/fnins.2011.00099
Huber, P., Kleiner, B., Gasser, T., and Dumermuth, G. (1971). Statistical methods for investigating phase relations in stationary stochastic processes. IEEE Trans. Acoust. 19, 78-86.

Huizenga, H., de Munck, J., Waldorp, L., Grasman, and R. P. (2002). Spatiotemporal EEG/MEG source analysis based on a parametric noise covariance model. IEEE Trans. Biomed. Eng. 49, 533-539.

Jerbi, K., Lachaux, J., N’Diaye, K., Pantazis, D., Leahy, R., Garnero, L., and Baillet, S. (2007). Coherent neural representation of hand speed in humans revealed by MEG imaging. Proc. Natl. Acad. Sci. U.S.A. 104, 7676-7681.

Kaper, M., Meinicke, P., Grossekathoefer, U., Lingner, T., and Ritter, H. (2004). BCI competition 2003 - data set IIb: support vector machines for the $\mathrm{P} 300$ speller paradigm. IEEE Trans. Biomed. Eng. 51, 1073-1076.

Koles, Z. J. (1991). The quantitative extraction and topographic mapping of the abnormal components in the clinical EEG. Electroencephalogr. Clin. Neurophysiol. 79, 440-447.

Krauledat, M., Shenoy, P., Blankertz, B., Rao, R. P. N., and Müller, K.-R. (2007). "Adaptation in CSPbased BCI systems," in Toward Brain-Computer Interfacing, eds G. Dornhege, R. Millán Jdel T. Hinterberger, D. McFarland, and K.R. Müller (Cambridge, MA: MIT Press), 305-309.

Kubanek, J., Miller, K., Ojemann, J., Wolpaw, J., and Schalk, G. (2009). Decoding flexion of individual fingers using electrocorticographic signals in humans. J. Neural Eng. 6 , 066001.

Kübler, A., Kotchoubey, B., Kaiser, J., Wolpaw, J., and Birbaumer, N. (2001). Brain-computer communication: unlocking the locked in. Psychol. Bull. 127, 358-375.

Leeb, R., Lee, F., Keinrath, C., Scherer R., Bischof, H., and Pfurtscheller, G. (2007). Brain-computer communication: motivation, aim and impact of exploring a virtual apartment. IEEE Trans. Neural Syst. Rehabil. Eng. $15,473-482$.

Lemm, S., Blankertz, B., Dickhaus, T. and Müller, K.-R. (2011). Introduction to machine learning for brain imaging. Neuroimage 56, 387-399.

Lemm, S., Schafer, C., and Curio, G. (2004). BCI competition 2003 - data set III: probabilistic modeling of sensorimotor mu rhythms for classification of imaginary hand movements. IEEE Trans. Biomed. Eng. 51, 1077-1080. 
Leuthardt, E., Schalk, G., Wolpaw, J., Ojemann, J., and Moran, D. (2004). A brain-computer interface using electrocorticographic signals in humans. J. Neural Eng. 1, 63-71.

Liang, N., and Bougrain, L. (2012). Decoding finger flexion from band-specific ECoG signals in humans. Front. Neurosci. 6:29. doi:10.3389/fnins.2012.00029

Liu, T., Goldberg, L., Gao, S., and Hong, B. (2010). An online brain-computer interface using non-flashing visual evoked potentials. J. Neural Eng. 7, 036003 .

Lotte, F., and Guan, C. (2011). Regularizing common spatial patterns to improve BCI designs: unified theory and new algorithms. IEEE Trans. Biomed. Eng. 58, 355-362.

Luo, A., and Sullivan, T. J. (2010). A user-friendly SSVEP-based braincomputer interface using a timedomain classifier. J. Neural Eng. 7, 26010.

Lv, J., Li, Y., and Gu, Z. (2010). Decoding hand movement velocity from electrocorticogram signals during a drawing task. Biomed. Eng. Online 9, $1-21$.

Mazaheri, A., and Jensen, O. (2008). Asymmetric amplitude modulations of brain oscillations generate slow evoked responses. J. Neurosci. 28, 7781-7787.

McFarland, D., Miner, L. A., Vaughan, T. M., and Wolpaw, J. (2000). Mu and beta rhythm topographies during motor imagery and actual movements. Brain Topogr. 12, 177-186.

Mehring, C., Rickert, J., Vaadia, E., Cardoso de Oliveira, S., Aertsen, A., and Rotter, S. (2003). Inference of hand movements from local field potentials in monkey motor cortex. Nat. Neurosci. 6, 1253-1254.

Mensh, B. D., Werfel, J., and Seung, H. S. (2004). BCI competition 2003 data set Ia: combining gamma-band power with slow cortical potentials to improve single-trial classification of electroencephalographic signals. IEEE Trans. Biomed. Eng. 51, 1052-1056.

Milekovic, T., Fischer, J., Pistohl, T., Ruescher, J., Schulze-Bonhage, A., Aertsen, A., Rickert, J., Ball, T., and Mehring, C. (2012). An online brainmachine interface using decoding of movement direction from the human electrocorticogram. J. Neural Eng. 9, 046003. doi: 10.1088/17412560/9/4/046003

Miller, K., Leuthardt, E., Schalk, G., Rao, R., Anderson, N., Moran, D., Miller, J., and Ojemann, J. (2007). Spectral changes in cortical surface potentials during motor movement. J. Neurosci. 27, 2424.

Miller, K., Schalk, G., Fetz, E., den Nijs, M., Ojemann, J., and Rao, R. (2010). Cortical activity during motor execution, motor imagery, and imagerybased online feedback. Proc. Natl. Acad. Sci. U.S.A. 107, 4430.

Miller, K., Sorensen, L., Ojemann, J., and Den Nijs, M. (2009a). Power-law scaling in the brain surface electric potential. PLoS Comput. Biol. 5, e1000609. doi:10.1371/journal.pcbi.1000609

Miller, K., Zanos, S., Fetz, E., den Nijs, M., and Ojemann, J. (2009b). Decoupling the cortical power spectrum reveals real-time representation of individual finger movements in humans. J. Neurosci. 29, 3132-3137.

Müller, K.-R., Tangermann, M., Dornhege, G., Krauledat, M., Curio, G., and Blankertz, B. (2008). Machine learning for real-time single-trial EEG-analysis: from brain-computer interfacing to mental state monitoring. J. Neurosci. Methods 167, 82-90.

Müller-Putz, G. R., Breitwieser, C., Tangermann, M., Schreuder, M., Tavella, M., Leeb, R., Cincotti, F., Leotta, F., and Neuper, C. (2011). Tobi hybrid BCI: principle of a new assistive method. Int. J. Bioelectromagn. 13, 144-145.

Naeem, M., Brunner, C., Leeb, R., Graimann, B., and Pfurtscheller, G. (2006). Seperability of four-class motor imagery data using independent components analysis. J. Neural Eng. 3, 208-216.

Nikulin, V. V., Linkenkaer-Hansen, K., Nolte, G., and Curio, G. (2010). Non-zero mean and asymmetry of neuronal oscillations have different implications for evoked responses. Clin. Neurophysiol. 121, 186-193.

Nikulin, V. V., Linkenkaer-Hansen, K., Nolte, G., Lemm, S., Müller, K.R., Ilmoniemi, R. J., and Curio, G. (2007). A novel mechanism for evoked responses in human brain. Eur. J. Neurosci. 25, 3146-3154.

Nikulin, V. V., Nolte, G., and Curio, G. (2011). A novel method for reliable and fast extraction of neuronal EEG/MEG oscillations on the basis of spatio-spectral decomposition. Neuroimage 55, 1528-1535.

Nolte, G., and Dassios, G. (2005). Analytic expansion of the EEG lead field for realistic volume conductors. Phys. Med. Biol. 50, 3807-3823.

Pfurtscheller, G. (1981). Central beta rhythm during sensorimotor activities in man. Electroencephalogr. Clin. Neurophysiol. 51, 253-264.
Pfurtscheller, G., Allison, B. Z., Bauernfeind, G., Brunner, C., Escalante, T. S., Scherer, R., Zander, T. O., Mueller-Putz, G., Neuper, C., and Birbaumer, N. (2010). The hybrid BCI. Front. Neurosci. 4:30. doi:10.3389/fnpro.2010.00003

Pfurtscheller, G., and da Silva, F. H. L. (1999). Event-related EEG/MEG synchronization and desynchronization: basic principles. Clin. Neurophysiol. 110, 1842-1857.

Pfurtscheller, G., and Lopes da Silva, F. (1999). "Functional meaning of event-related desynchronization (ERD) and synchronization (ERS)," in Event-Related Desynchronization. Handbook of Electroencephalography and Clinical Neurophysiology, Vol. 6 eds G. Pfurtscheller and F. Lopes da Silva (Amsterdam: Elsevier), 51-66.

Pfurtscheller, G., Stancak, A. Jr., and Neuper, C. (1996). Event-related synchronization (ERS) in the alpha band-an electrophysiological correlate of cortical idling: a review. Int. J. Psychophysiol. 24, 39-46.

Pistohl, T., Ball, T., Schulze-Bonhage, A., Aertsen, A., and Mehring, C. (2008). Prediction of arm movement trajectories from ECoG-recordings in humans. J. Neurosci. Methods 167, 105-114.

Popescu, F., Fazli, S., Badower, Y., Blankertz, B., and Müller, K.-R. (2007). Single trial classification of motor imagination using 6 dry EEG electrodes. PLoS ONE 2, e637. doi:10.1371/journal.pone.0000637

Porbadnigk, A. K., Antons, J.-N., Blankertz, B., Treder, M. S., Schleicher, R., Möller, S., and Curio, G. (2010). Using ERPs for assessing the (sub)conscious perception of noise. Conf. Proc. IEEE Eng. Med. Biol. Soc 2010, 2690-2693.

Porbadnigk, A. K., Scholler, S. Blankertz, B., Ritz, A., Born, M., Scholl, R., Müller, K.-R., Curio, G., and Treder, M. S. (2011). Revealing the neural response to imperceptible peripheral flicker with machine learning. Conf. Proc. IEEE Eng. Med. Biol. Soc. 2011, 3692-3695.

Quek, M., Boland, D., Williamson, J., Murray-Smith, R., Tavella, M., Perdikis, S., Schreuder, M., and Tangermann, M. (2011). "Simulating the feel of brain-computer interfaces for design, development and social interaction," in Proceedings of the 2011 Annual Conference on Human Factors in Computing Systems, CHI '11, New York, NY: ACM, 25-28.

Rakotomamonjy, A., and Guigue, V. (2008). BCI competition III: dataset
II- ensemble of SVMs for BCI P300 speller. IEEE Trans. Biomed. Eng. 55, 1147-1154.

Ramoser, H., Müller-Gerking, J., and Pfurtscheller, G. (2000). Optimal spatial filtering of single trial EEG during imagined hand movement. IEEE Trans. Rehabil. Eng. 8, 441-446.

Ramsey, L., Tangermann, M., Haufe, S., and Blankertz, B. (2009). Practicing fast-decision BCI using a "goalkeeper" paradigm. BMC Neurosci. 10(Suppl. 1), P69. doi:10.1186/14712202-10-S1-P69

Rickert, J., Cardoso de Oliveira, S. Vaadia, E., Aertsen, A., Rotter, S., and Mehring, C. (2005). Encoding of movement direction in differen frequency ranges of motor cortical local field potentials. J. Neurosci. 25, 8815-8824.

Saab, J., Battes, B., and GrosseWentrup, M. (2011). Simultaneous EEG Recordings with Dry and Wet elecTrodes in Motor-Imagery. Graz: Verlag der Technischen Universität Graz, 312-315.

Sajda, P., Gerson, A., Müller, K.-R., Blankertz, B., and Parra, L. (2003). A data analysis competition to evaluate machine learning algorithms for use in brain-computer interfaces. IEEE Trans. Neural Syst. Rehabil. Eng. 11, 184-185.

Sannelli, C., Braun, M., Tangermann, M., and Müller, K.-R. (2008). "Estimating noise and dimensionality in BCI data sets: towards BCI illiteracy comprehension," in Proceedings of the 4th International BrainComputer Interface Workshop and Training Course 2008, Verlag der Technischen Universität Graz, Graz, 26-31.

Sannelli, C., Vidaurre, C., Müller, K.-R., and Blankertz, B. (2011). Common spatial pattern patches - an optimized filter ensemble for adaptive brain-computer interfaces. J. Neural Eng. 8, 025012.

Sardouie, S. H., and Shamsollahi, M. B. (2012). Discriminating meg signals recorded during hand movements using selection of efficient features. Front. Neurosci. 6:42. doi:10.3389/fnins.2012.00042

Schaeff, S., Treder, M., Venthur, B., and Blankertz, B. (2011). Motion-based ERP spellers in a covert attention paradigm. Neurosci. Lett. 500, e11.

Schalk, G., Kubánek, J., Miller, K., Anderson, N., Leuthardt, E., Ojemann, J., Limbrick, D., Moran, D., Gerhardt, L., and Wolpaw, J. (2007). Decoding two-dimensional movement trajectories using electrocorticographic signals in humans. $J$. Neural Eng. 4, 264-275. 
Schalk, G., McFarland, D., Hinterberger, T., Birbaumer, N., and Wolpaw, J. (2004). BCI2000: a general-purpose brain-computer interface (BCI) system. IEEE Trans. Biomed. Eng. 51, 1034-1043.

Schalk, G., Miller, K., Anderson, N., Wilson, J., Smyth, M., Ojemann, J., Moran, D., Wolpaw, J., and Leuthardt, E. (2008). Twodimensional movement control using electrocorticographic signals in humans. J. Neural Eng. 5, 75 .

Schlögl, A., Keinrath, C., Zimmermann, D., Scherer, R., Leeb, R., and Pfurtscheller, G. (2007a). A fully automated correction method of EOG artifacts in EEG recordings. Clin. Neurophysiol. 118, 98-104.

Schlögl, A., Kronegg, J., Huggins, J., and Mason, S. (2007b). "Evaluation criteria in BCI research," in Toward Brain-Computer Interfacing, Chapt. 19, eds G. Dornhege, J. Millán, T. Hinterberger, D. J. McFarland, and K.-R. Müller (Cambridge: MIT Press), 327-342.

Schreuder, M., Blankertz, B., and Tangermann, M. (2010). A new auditory multi-class braincomputer interface paradigm: spatial hearing as an informative cue. PLoS ONE 5, e9813. doi:10.1371/journal.pone.0009813

Schreuder, M., Rost, T., and Tangermann, M. (2011). Listen, you are writing! Speeding up online spelling with a dynamic auditory BCI. Front. Neurosci. 5:112. doi:10.3389/fnins.2011.00112

Schröder, M., Bogdan, M., Rosenstiel, W., Hinterberger, T., and Birbaumer, N. (2003). "Automated EEG feature selection for brain computer interfaces," in Proceedings of the First International IEEE EMBS Conference on Neural Engineering, Capri, 626-629.

Shenoy, P., Krauledat, M., Blankertz, B., Rao, R. P. N., and Müller, K.R. (2006). Towards adaptive classification for BCI. J. Neural Eng. 3, R13-R23.

Silvoni, S., Ramos-Murguialday, A., Cavinato, M., Volpato, C., Cisotto, G., Turolla, A., Piccione, F., and Birbaumer, N. (2011). Brain-computer interface in stroke: a review of progress. Clin. EEG Neurosci. 42, 245-252.

Simons, R., Miller, G., Weerts, T., and Lang, P. J. (1981). Correcting baseline drift artifact in slow potential recording. Psychophysiology 19, 691-700.

Sugiyama, M., Krauledat, M., and Müller, K.-R. (2007). Covariate shift adaptation by importance weighted cross validation. J. Mach. Learn. Res. 8, 1027-1061.

Tangermann, M., Schreuder, M., Dähne, S., Höhne, J., Regler, S., Ramsay, A., Quek, M., Williamson, J., and Murray-Smith, R. (2011). Optimized stimulation events for a visual ERP BCI. Int. J. Bioelectromagn. 13, 119-120.

Treder, M. S., Schmidt, N. M., and Blankertz, B. (2011). Gazeindependent brain-computer interfaces based on covert attention and feature attention. J. Neural Eng. 8, 066003.

Velliste, M., Perel, S., Spalding, M., Whitford, A., and Schwartz, A. (2008). Cortical control of a prosthetic arm for self-feeding. Nature 453, 1098-1101.

Vidaurre, C., Sannelli, C., Müller, K.R., and Blankertz, B. (2011). Coadaptive calibration to improve BCI efficiency. J. Neural Eng. 8, 025009.

Vidaurre, C., and Schlögl, A. (2008). "Comparison of adaptive features with linear discriminant classifier for Brain Computer Interfaces," in Proceedings of the 30th Annual International Conference of the IEEE Engineering in Medicine and Biology Society 2008, 173-176.

von Bünau, P., Meinecke, F. C., Király, F., and Müller, K.-R. (2009). Finding stationary subspaces in multivariate time series. Phys. Rev. Lett. 103, 214101.

Waldert, S., Pistohl, T., Braun, C., Ball, T., Aertsen, A., and Mehring, C. (2009). A review on directional information in neural signals for brain-machine interfaces. J. Physiol. Paris 103, 244-254.

Waldert, S., Preissl, H., Demandt, E., Braun, C., Birbaumer, N., Aertsen, A., and Mehring, C. (2008). Hand movement direction decoded from
MEG and EEG. J. Neurosci. 28, 1000-1008.

Wang, W., Sudre, G. P., Xu, Y., Kass, R. E., Collinger, J. L., Degenhart, A. D., Bagic, A. I., and Weber D. J. (2010). Decoding and cortical source localization for intended movement direction with MEG. J. Neurophysiol. 104, 2451-2461.

Wang, Y., and Makeig, S. (2010). Predicting intended movement direction using EEG from human posterior parietal cortex. Lect. Notes Artif. Int. 5638, 437-446.

Wang, Y., Zhang, Z., Li, Y., Gao, X., Gao, S., and Yang, F. (2004). BCI competition 2003 - data set IV: an algorithm based on CSSD and FDA for classifying single-trial EEG. IEEE Trans. Biomed. Eng. 51, 1081-1086.

Wei, Q., Gao, X., and Gao, S. (2006). Feature extraction and subset selection for classifying single-trial ECoG during motor imagery. Conf. Proc. IEEE Eng. Med. Biol. Soc. 1, 1589-1592.

Winkler, I., Haufe, S., and Tangermann, M. (2011). Automatic classification of artifactual ICA-components for artifact removal in EEG signals. Behav. Brain Funct. 7, 30.

Witte, M., Galan, F., Waldert, S., Aertsen, A., Rotter, S., Birbaumer, N., Braun, C., and Mehring, C. (2010). "An online BCI system using hand movement recognition from MEG," in 4th International BCI Meeting 2010, Asilomar.

Wojcikiewicz, W., Vidaurre, C., and Kawanabe, M. (2011). "Stationary common spatial patterns: towards robust classification of non-stationary eeg signals," in Acoustics, Speech and Signal Processing (ICASSP), 2011 IEEE International Conference on Prague, 577-580.

Wolpaw, J. R., Birbaumer, N., McFarland, D. J., Pfurtscheller, G., and Vaughan, T. M. (2002). Braincomputer interfaces for communication and control. Clin. Neurophysiol. 113, 767-791.

Xu, N., Gao, X., Hong, B., Miao, X., Gao, S., and Yang, F. (2004). BCI competition 2003 - data set IIb: enhancing P300 wave detection using ICAbased subspace projections for $\mathrm{BCI}$ applications. IEEE Trans. Biomed. Eng. 51, 1067-1072.
Zander, T. O., Lehne, M., Ihme, K., Jatzev, S., Correia, J., Kothe, C., Picht, B., and Nijboer, F. (2011). A dry EEG-system for scientific research and brain-computer interfaces. Front. Neurosci. 5:53. doi:10.3389/fnins.2011.00053

Zhang, D., Wang, Y., Gao, X., Hong, B., and Gao, S. (2007). An algorithm for idle-state detection in motor-imagery-based braincomputer interface. Comput. Intell Neurosci. 2007, 39714. doi: 10.1155/2007/39714

Zhang, H., Guan, C., Ang, K. K., and Chin, Z. Y. (2012). Learning discriminative patterns for selfpaced EEG-based motor imagery detection. Front. Neurosci. 6:7. doi:10.3389/fnins.2012.00007

Conflict of Interest Statement: The authors declare that the research was conducted in the absence of any commercial or financial relationships that could be construed as a potential conflict of interest.

Received: 17 December 2011; paper pending published: 15 January 2012; accepted: 30 March 2012; published online: 13 July 2012.

Citation: Tangermann M, Müller K-R, Aertsen A, Birbaumer N, Braun C, Brunner $C$, Leeb $R$, Mehring $C$, Miller KJ, Müller-Putz GR, Nolte G, Pfurtscheller $G$, Preissl H, Schalk G, Schlögl A, Vidaurre $C$, Waldert $S$ and Blankertz $B$ (2012) Review of the BCI competition IV. Front. Neurosci. 6:55. doi: 10.3389/fnins.2012.00055

This article was submitted to Frontiers in Neuroprosthetics, a specialty of Frontiers in Neuroscience.

Copyright (C) 2012 Tangermann, Müller, Aertsen, Birbaumer, Braun, Brunner, Leeb, Mehring, Miller, MüllerPutz, Nolte, Pfurtscheller, Preissl, Schalk, Schlögl, Vidaurre, Waldert and Blankertz. This is an open-access article distributed under the terms of the Creative Commons Attribution License, which permits use, distribution and reproduction in other forums, provided the original authors and source are credited and subject to any copyright notices concerning any third-party graphics etc. 


\section{A. APPENDIX}

\section{A.1. DATA SET 2A}

All files are listed in Table A1. Note that the test sets will be made available after the deadline of the competition (except for one file from subject A01, which serves as an example). The GDF files can be loaded using the open-source toolbox BioSig, available for free at http://biosig.sourceforge.net/. There are versions for Octave $^{1} /$ FreeMat $^{2} / \mathrm{MATLAB}^{3}$ as well as a library for $\mathrm{C} / \mathrm{C}++$.

A GDF file can be loaded with the BioSig toolbox with the following command in Octave/FreeMat/MATLAB (for $\mathrm{C} / \mathrm{C}++$, the corresponding function HDRTYPE* sopen and size_t sread must be called):

$$
[\mathrm{S}, \mathrm{h}]=\operatorname{sload}\left({ }^{\prime} \mathrm{A} 01 \mathrm{~T} \cdot \mathrm{gdf} \mathrm{f}^{\prime}\right) ;
$$

Note that the runs are separated by 100 missing values, which are encoded as not-a-numbers $(\mathrm{NaN})$ by default. Alternatively, this behavior can be turned off and the missing values will be encoded as the negative maximum values as stored in the file with:

${ }^{1}$ http://www.gnu.org/software/octave/

${ }^{2}$ http://freemat.sourceforge.net/

${ }^{3}$ The MathWorks, Inc., Natick, MA, USA

Table A1 | (Data Set 2a). List of all files contained in the data set 2a, the striked out test data sets were provided only after the deadline of the competition.

\begin{tabular}{lll}
\hline ID & Training & Test \\
\hline 1 & A01T.gdf & A01E.gdf \\
2 & A02T.gdf & A02E.gdf \\
3 & A03T.gdf & A03E.gdf \\
4 & A04T.gdf & A04E.gdf \\
5 & A05T.gdf & A05E.gdf \\
6 & A06T.gdf & A06E.gdf \\
7 & A07T.gdf & A07E.gdf \\
8 & A08T.gdf & A08E.gdf \\
9 & A09T.gdf & A09E.gdf
\end{tabular}

Note that due to technical problems the EOG block is shorter for subject AO4T and contains only the eye movement condition.

Table A2 | (Data Set 2a). List of event types in data set 2a (the first column contains decimal values and the second hexadecimal values).

\begin{tabular}{rll}
\hline \multicolumn{2}{c}{ Event type } & Description \\
\hline 276 & $0 \times 0114$ & Idling EEG (eyes open) \\
277 & $0 \times 0115$ & Idling EEG (eyes closed) \\
768 & $0 \times 0300$ & Start of a trial \\
769 & $0 \times 0301$ & Cue onset left (class 1) \\
770 & $0 \times 0302$ & Cue onset right (class 2) \\
771 & $0 \times 0303$ & Cue onset foot (class 3) \\
772 & $0 \times 0304$ & Cue onset tongue (class 4) \\
783 & $0 \times 030 \mathrm{~F}$ & Cue unknown \\
1023 & $0 \times 03 \mathrm{FF}$ & Rejected trial \\
1072 & $0 \times 0430$ & Eye movements \\
32766 & $0 \times 7 \mathrm{FFE}$ & Start of a new run
\end{tabular}

$[s, h]=\operatorname{sload}\left({ }^{\prime} A 01 T \cdot g d f ', 0\right.$,

' OVERFLOWDETECTION : OFF' ) ;

The workspace will then contain two variables, namely the signals $s$ and a header structure $h$. The signal variable contains 25 channels (the first 22 are EEG and the last 3 are EOG signals). The header structure contains event information that describes the structure of the data over time. The following fields provide important information for the evaluation of this data set:

\section{h.EVENT . TYP \\ h.EVENT.POS \\ h.EVENT.DUR}

The position of an event in samples is contained in h.EVENT.POS. The corresponding type can be found in h. EVENT. TYP, and the duration of that particular event is stored in $\mathrm{h}$. EVENT . DUR. The types used in this data set are described in Table A2 (hexadecimal values, decimal notation in parentheses). Note that the class labels (i.e., 1, 2, 3, 4 corresponding to event types $769,770,771,772$ ) are only provided for the training data and not for the testing data.

The trials containing artifacts as scored by experts are marked as events with the type 1023. In addition, $\mathrm{h}$. Artifact Selection contains a list of all trials, with 0 corresponding to a clean trial and 1 corresponding to a trial containing an artifact.

SigViewer 0.2 (or higher) can be used to view and annotate GDF files. SigViewer is available at http://sigviewer.sourceforge.net/.

\section{A.2. DATA SET 2B}

All files are listed in Table A3. The GDF files can be loaded using the open-source toolbox BioSig, available for

Table A3 | (Data Set 2b). List of all files contained in the data set 2b, the striked out test data sets will be provided after the deadline of the competition.

\begin{tabular}{lll}
\hline ID & Training & Test \\
\hline 1 & B0101T, B0102T, B0103T & B0104E, B0105E \\
2 & B0201T, B0202T, B0203T & B0204E, B0205E \\
3 & B0301T, B0302T, B0303T & B0304E, B0305E \\
4 & B0401T, B0402T, B0403T & B0404E, B0405E \\
5 & B0501T, B0502T, B0503T & B0504E, B0505E \\
6 & B0601T, B0602T, B0603T & B0604E, B0605E \\
7 & B0701T, B0702T, B0703T & B0704E, B0705E \\
8 & B0801T, B0802T, B0803T & B0804E, B0805E \\
9 & B0901T, B0902T, B0903T & B0904E, B0905E \\
\hline The first two sessions (...01T, ...02T) contain training data without feedback, and \\
the last three sessions (...03T, ...04E, ...05E) with smiley feedback. Note: Due \\
to technical problems no recording for EOG estimation (eyes open, closed, eye \\
movements) exists in session B0102T and B0504E.
\end{tabular}


free at http://biosig.sourceforge.net/. There are versions for Octave $^{4} / \mathrm{MATLAB}^{5}$ as well as a library for $\mathrm{C} / \mathrm{C}++$.

A GDF file can be loaded with the BioSig toolbox with the following command in Octave/MATLAB (for $\mathrm{C} / \mathrm{C}++$, the corresponding function HDRTYPE* sopen and size_t sread must be called):

$$
[\mathrm{s}, \mathrm{h}]=\operatorname{sload}\left({ }^{\prime} \mathrm{B} 0101 \mathrm{~T} \cdot \mathrm{gdf} \mathrm{f}^{\prime}\right) ;
$$

Note that the runs are separated by 100 missing values, which are encoded as not-a-numbers ( $\mathrm{NaN}$ ) by default. Alternatively, this behavior can be turned off and the missing values will be encoded as the negative maximum values as stored in the file with:

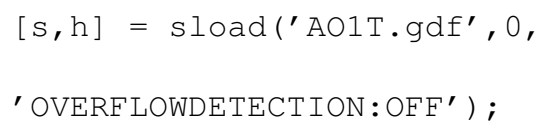

The workspace will then contain two variables, namely the signals $s$ and the header structure $h$. The signal variable contains 6 channels (the first 3 are EEG and the last 3 are EOG signals). The header structure contains event information that describes the structure of the data over time. The following fields provide important information for the evaluation of this data set:

$$
\begin{aligned}
& \text { h. EVENT } . \text { TYP } \\
& \text { h.EVENT.POS } \\
& \text { h.EVENT.DUR }
\end{aligned}
$$

The position of an event in samples is contained in h.EVENT.POS. The corresponding type can be found in

${ }^{4}$ http://www.gnu.org/software/octave/

${ }^{5}$ The MathWorks, Inc., Natick, MA, USA
Table A4 | (Data Set 2b). List of event types in data set 2b (the first column contains decimal values and the second hexadecimal values).

\begin{tabular}{rll}
\hline \multicolumn{1}{c}{ Event type } & Description \\
\hline 276 & $0 \times 0114$ & Idling EEG (eyes open) \\
277 & $0 \times 0115$ & Idling EEG (eyes closed) \\
768 & $0 \times 0300$ & Start of a trial \\
769 & $0 \times 0301$ & Cue onset left (class 1) \\
770 & $0 \times 0302$ & Cue onset right (class 2) \\
781 & $0 \times 030 \mathrm{D}$ & BCl feedback (continuous) \\
783 & $0 \times 030 \mathrm{~F}$ & Cue unknown \\
1023 & $0 \times 03 \mathrm{FF}$ & Rejected trial \\
1077 & $0 \times 0435$ & Horizontal eye movement \\
1078 & $0 \times 0436$ & Vertical eye movement \\
1079 & $0 \times 0437$ & Eye rotation \\
1081 & $0 \times 0439$ & Eye blinks \\
32766 & $0 \times 7 F F E$ & Start of a new run \\
\hline
\end{tabular}

h.EVENT . TYP, and the duration of that particular event is stored in $\mathrm{h}$. EVENT.DUR. The types used in this data set are described in Table A4 (hexadecimal values, decimal notation in parentheses). Note that the class labels (i.e., 1 and 2, corresponding to event types 769 and 770) are only provided for the training data and not for the testing data.

The trials containing artifacts as scored by experts are marked as events with the type 1023. In addition, h. Artifact Selection contains a list of all trials, with 0 corresponding to a clean trial and 1 corresponding to a trial containing an artifact.

In order to view the GDF files, the viewing and scoring application SigViewer v0.2 or higher (part of BioSig) can be used. 